\title{
Online Auction and List Price Revenue Management
}

\author{
René Caldentey Gustavo Vulcano *
}

April 13, 2004

\begin{abstract}
We analyze a revenue management problem in which a seller facing a Poisson arriving stream of customers operates an online multiunit auction. Customers have an alternative list price channel where to get the product from. We consider two variants of this problem: In the first one, the list price is an external channel run by another firm. In the second variant, the seller manages simultaneously both the auction and the list price channels.

Each consumer, trying to maximize his own surplus, must decide either to buy at the posted price and get the item at no risk, or to join the auction and wait until its end, where the winners are revealed and the auction price is disclosed.

Our approach consists of two parts. First, we study structural properties of the problem, and show that the equilibrium strategy for both versions of this game is of the threshold type, meaning that a consumer will join the auction only if his arrival time is above a function of his own valuation. This consumer's strategy can be computed using an iterative algorithm in a function space, provably convergent under some conditions. Unfortunately, this procedure is computationally intensive.

To overcome this, we formulate an asymptotic version of the problem, in which the demand rate and the initial number of units grow proportionally large. We get a simple closed form for the equilibrium strategy in this regime, which is then used as an approximated solution for the original problem. Numerical computations show that this heuristic is very accurate. The asymptotic solution culminates then in simple and precise recipes for how bidders should behave, and how the seller should structure the auction, and price the product in the dual channel case.
\end{abstract}

Key words: revenue management, online auction, dual channel, strategic behavior, asymptotic analysis

\footnotetext{
${ }^{*}$ Both authors are with the Stern School of Business, New York University, New York. E-Mails: \{rcaldent, gvulcano\}@stern.nyu.edu.
} 


\section{Introduction}

Revenue management (RM) is viewed by many as one of the most important management sciences and operations research practices (see Bell [2]). Traditionally, it has involved setting list prices and controlling a fixed capacity to maximize revenues, achieving a high impact in revenue performance of the airline, hospitality, railway and car rental industries. RM has then extended its domain to the broader area of dynamic pricing, with applications in the retail industry. (See Talluri and van Ryzin [32] for a description of RM methods and applications, or the survey by Bitran and Caldentey [5] for an overview of dynamic pricing models).

In the last few years though, RM has widened its focus to alternative mechanisms for pricing proposed by electronic commerce, such as guaranteed purchase contracts, group purchasing, online negotiations and auctions. (See Boyd and Bilegan [6]) for a survey of revenue management and ecommerce, mainly oriented to the airline industry.) Although list pricing is probably still the most familiar and used pricing mechanism, online auctions are an increasing phenomenon, easy to verify in a quick Internet navigation. Klein [16] and Klein and O'Keefe [17] mention the expansion to more participants, the reduced transaction costs for both buyers and sellers, and the ability to conduct complex auctions as the main factors that has determined the dramatic impact that Internet has had on auctions. In a recent and complete survey about online auctions, Pinker et al. [28] add two more factors in this direction: easy collection of data about auctions, and the possibility for participants to join at any time.

Nowadays, the same product is sold simultaneously through posted price and auction channels. For example, airline and cruise companies sell tickets through their own websites at posted prices, but also through auctions run by Priceline.com; IBM and Sun Microsystems sell their products at their own website, but also offer some selected new and refurbished products through eBay.com auctions. Sony and Hewlett Packard use uBid.com auctions to dispose excess inventory. SharperImage.com sells electronics and home appliances at posted prices, but also offer new, refurbished and less-than-new products through auctions at the own site and at eBay. OnSale.com also hosts the auction channel of many firms, offering a huge variety of products.

These trends raise some new important theoretical and practical questions. In particular, this paper focuses on analyzing the strategic behavior of the consumers, and on how the seller should manage online auctions, and parallel online auction and list price channels with the objective of maximizing revenues. A simple observation is that any person with an Internet connection becomes a potential buyer and is just a click away of a commercial transaction. Therefore, the consumer's ability of checking prices and bid states in different channels reinforces his strategic behavior at the time of deciding whether to buy at a posted price or to join an auction. The dependence between the two channels becomes clear, and should not be ignored by the seller when designing a business to globally maximize revenues. On top of that, particularly in the business-to-consumer (B2C) market, the seller faces an additional challenge in the dual channel case: She does not want to offer a business model 
that cannibalizes by itself. When offering a high posted price, she narrows the list price channel, and middle-to-high value customers are tempted to join the auction, which could eventually decrease revenues. On the other hand, if she posts a low price, she widens the list price channel by attracting high value customers who leave money on the table.

Our purpose is shading some light on this tradeoff. We analyze a single period model in which a seller operates an online auction, offering multiple units of an homogeneous good. The seller announces the initial capacity $Q_{0}$, the auction duration $T$, and the auction reservation price $v^{\mathrm{R}}$. The type of auction that the seller conducts in both cases is a multiunit second-price auction. Buyers with single unit demand arrive according to a Poisson process. They have a private value for the product. These values are independent and identically distributed, following a continuous distribution that is common knowledge. They must decide whether to bid and wait for the auction outcome, or buy at the posted price and get the unit instantaneously. We consider two variations of this problem:

- In the single auction channel case, the external fixed price is run by another firm, which we assume has unlimited capacity. If a bidder is not among the winners, he can buy the item at the posted price at later time $T$, though his utility is discounted.

- In the dual auction and list price channels, the seller is a monopolist that also announces the posted price $\hat{P}$. Here, joining the auction from the bidders' perspective means running the risk of no auction occurring at time $T$, provided all the items were cleared through the fixed price channel. We assume that the arriving customers are informed about the initial number of units offered, even though this quantity has already been depleted during the elapsed time through the list price channel.

The buyer's strategy consists of two dimensions: deciding whether to join the auction or not, and in case of joining, what to bid. The supply size under both scenarios produces different bidding behaviors. The infinite availability in the first case induces no bidder to bid higher than the posted price: In case he loses, he always has the chance to pay that price at moment $T$. In the dual channel, the finite supply induces the standard dominant "bid your own value" strategy for second-price auctions (See Krishna [19, Section 2.2] for a detailed analysis of bidding behavior under a standard second-price auction).

Regarding the participation decision, we argue that in the game taking place among costumers themselves, and between them and the seller (for both settings described above), the symmetric equilibrium strategy is defined by a threshold function in the space (valuation, time): For a customer arriving at time $t$ with valuation $v$, there is a threshold $H(v)$ such that if $H(v) \leq t$, then he will participate in the auction. Otherwise, he will buy at the posted price $\hat{P}$. We prove the existence of this threshold function, and characterize some properties of it.

This consumer's strategy can be computed using an iterative algorithm in a function space, provably convergent under some special conditions. Unfortunately, this procedure is computationally 
intensive, as is usually the case. To overcome this, we formulate an asymptotic version of the problem, in which the demand rate and the initial number of units grow proportionally large. We get a simple closed form for the equilibrium strategy in this limiting regime, which is then used as an approximated solution for the original problem. Numerical computations show that this heuristic is very accurate. We can assert then that the asymptotic solution culminates in precise and simple recipes for how bidders should behave and how the seller should structure the auction, and price the product for the dual channel case.

Finally, we analyze the seller's optimization problem in each of the settings, and using the asymptotic participation strategy of the consumers, we find numerically the optimal reservation price (and list price for the dual channel) that the seller must announce to maximize revenues.

\subsection{Literature review}

Auctions have been extensively studied in the economic literature (e.g. see the seminal work of Vickrey [36], the influential paper of Milgrom and Weber [25], the survey by Klemperer [18], or the recent book by Krishna [19]). Price discrimination has been argued as one of the main reasons for using them (see Bulow and Roberts [8]). Maskin and Riley [24] proved the optimality of the second-price mechanism for the single period multiunit auction.

Several papers have put auctions in an operational perspective, mainly focusing on mechanism design approaches. Regarding its connection with RM, Cooper and Menich [10] proposed a procedure to auction airline tickets on a network of flights. Pinker et al. [27] study how to run a sequence of standard multiunit auctions, using bidding information to learn about the customer valuation distribution. Vulcano et al. [37] analyze an optimal dynamic auction for a firm selling a fixed capacity over a finite horizon, and show how to set reservation prices for each of the units in different periods.

The firm's choice between auctions and posted prices, but restricted to the purpose of managing a single channel, has also been addressed (e.g. see De Vany [35], Wang [38], Harstad [15], etc.) Gallien [13] presents a mechanism design study, where a seller faces a renewal process of customers with single unit demand. He reduces the continuous problem to a discrete time infinite horizon dynamic program, and proves that the optimal solution consists of a sequence of increasing posted prices.

There are some papers presenting overviews of both consumer-to-consumer $(\mathrm{C} 2 \mathrm{C})$ and B2C frameworks (e.g. see Beam and Segev [1] and Lucking-Reiley [22]). They find that the auction mechanisms used in the Internet are mostly the traditional ones (mainly English, Dutch, and first-price sealed-bid).

The problem of jointly managing auction and list price channels has not been deserved much attention in the literature though. New features like the buy now prices has been recently addressed by Budish and Takeyama [7], although their model is limited to two bidders and two valuation types.

Within the B2C framework, the empirical study of Vakrat and Seidmann [33] compares prices paid through on-line auctions and catalogs for the same product. They observe that auctions result 
in average prices $25 \%$ below the catalog ones. They build a simple model of single unit auctions with deterministic number of bidders, but ignoring customer choice behavior. In the infinite horizon model of van Ryzin and Vulcano [34, Section 3.3], the seller operates simultaneously auctions and posted prices, and replenishes her stock in every period. However, the streams of consumers for both channels are independent, and the seller decides how many units to allocate to each of the channels.

Our research is mainly motivated by the work of Etzion et al. [12]. They also analyze simultaneous dual online auctions and list price channels in a B2C framework, with customers arriving according to a Poisson process, and deciding which channel to join. We find their work interesting and insightful. There are important differences between the models though that we point out here.

First, Etzion et al. [12] optimize the seller's average expected revenue per unit of time, over a repeated sequence of games. Their decision variables are the auction length, the quantity to auction in each period, and the price to post. They assume that the seller capacity is unlimited, and that losers in the auction can get the item in the other channel at the end of each period, with probability one. They found two optimal auction design strategies: short single unit auctions and long multi-unit auctions. In our dual-channel model, we instead assume that the seller's capacity is limited, and her decision is how many units to offer simultaneously to both channels. Hence, bidders run the risk of not getting the object in case the total capacity is depleted before time $T$.

Secondly, Etzion et al. [12] characterize the customer's equilibrium strategy with a single value $\bar{t}$, such that all consumers with valuation below the posted price, and those arriving later than the threshold $\bar{t}$ with valuation above the posted price, will join the auction. All other customers will go to the online catalog. Our equilibrium participation strategy turns out to be more complex, and is defined by a continuous threshold function in the space (valuation, time). Also, when computing the participation strategy in their paper, consumers assume that the total number of them is deterministically set at the mean of the Poisson distribution. We instead embed the random nature of the arrival process in the computation of the customer's participation strategy. A distinguished characteristic of our research is the asymptotic analysis of the game. We show that the complex threshold that describes the strategic behavior of the consumers can be easily computed in the limiting regime where the customers' arrival rate and the number of units offered grow proportionally large.

Overall, we believe that the two models share some features, but contrast in some important dimensions that are worth exploring.

Finally, the problem of analyzing the equilibrium of a system where customers arrive during a time window has been addressed by few papers, though they are oriented to the characterization of the arrival pattern (e.g. see Glazer and Hassin [14] or Lariviere and Van Mieghem [20]). In our setting, the arrival process is exogenous, and we concentrate on characterizing the Nash equilibrium (in pure strategies) of the participation behavior of the customers. 


\subsection{Organization of this paper}

The remainder of this paper is organized as follows: We introduce the model for both variants of the problem in Section 2. In Section 3 we focus on the single auction channel case, characterizing the symmetric participation equilibrium of the consumers, and presenting its asymptotic analysis. The seller's optimization problem is introduced in subsection 3.4. An extension of this study for the dual channel with static list price is described in Section 4. Section 5 has our concluding remarks. All the proofs are in the Appendix.

\section{Model description}

We study the problem faced by a firm (seller) offering $Q_{0}$ units of an homogeneous product through an online auction with reservation price $v^{\mathrm{R}}$, during a fixed planning horizon of length $T$.

Customers have single unit demand, and visit the seller's website following a Poisson process with constant intensity $\lambda$. They are characterized by two quantities: ( $i)$ their arrival time, and $(i i)$ their private valuation for the product. For notational convenience, we denote by $v_{t}$ the private valuation of a buyer arriving at time $t$. Note that this notation is well-defined since with probability one the Poisson process has at most one arrival at a given time. We also assume that the cumulative probability distribution $F$ of the random variable $v_{t}$ is a time homogeneous and differentiable function with support $\mathcal{V} \triangleq[0, \bar{v}]$ that admits a density function $f(v)$. Both $\lambda$ and $F$ are common knowledge. Without loss of generality, we assume from now on that $\bar{v}=1$, that is, we scale all prices in this economy by $\bar{v}$.

When visiting the website, the buyers must choose either to bid or to buy the product at a posted price $\hat{P}$. We model buyers' purchasing decision depending on their private valuation as well as on the time elapsed until they are likely to get the object. Specifically, we assume that buyers are sensitive to delay. We denote by $u(t, \tau, v-p)$ the discounted utility perceived by a buyer arriving at time $t$ with valuation $v$ who eventually gets a unit of product at time $\tau$ at a price $p$ (paid at the moment of getting it). If the buyer never gets the object we use the convention $\tau=\infty$. We consider an exponentially discounted utility function of the form:

$$
u(t, \tau, v-p)=(v-p) \exp (-w(\tau-t))
$$

where $w$ is a fixed constant shared by all buyers. However, our main theoretical results are not particularly tight to the functional form of the utility in (1) as long as it remains increasing in $v-p$ and decreasing in delay $\tau-t .^{1}$

We assume that the seller and the buyers are risk neutral, and present two variants of this problem. In the first one, the seller manages a single auction channel, and there is an external market with infinite

\footnotetext{
${ }^{1}$ Note that the buyer's utility function is of the intertemporal type (e.g. see Mas-Colell et.al. [23, Chapter 20]).
} 
supply where the same product is offered at a fixed price $\hat{P}$. Arriving buyers must choose between either buying at the posted price (and getting the item with probability one), or bidding in the auction (and eventually being awarded the item at time $T$ ). If a bidder is not among the winners, he can still buy the item at the posted price at time $T$, though his utility will be discounted according to (1).

In the second variant of the problem, the seller is a monopolist managing simultaneously both the auction and the list price channels. In this case, the seller's capacity is depleted during the time horizon, when she allocates units through the list price channel. Therefore, the number of units to be auctioned is described by a random variable $Q_{T}$, with support $\left\{0, \ldots, Q_{0}\right\}$, meaning that the customer is uncertain about the number of units to be auctioned. We also assume that in the website, the seller announces the time left for the auction completion, the initial number of units $Q_{0}$, the reservation price $v^{\mathrm{R}}$, and the posted price $\hat{P}$.

For both settings, by denoting $Q_{T}$ the number of units auctioned at time $T$, we model the auction as a sealed-bid multiunit second-price (also referred to as $\left(Q_{T}+1\right)$-price) auction, with reservation price $v^{\mathrm{R}}$. In a $\left(Q_{T}+1\right)$-price auction, the winners are the $Q_{T}$ highest bidders in excess of $v^{\mathrm{R}}$, and all of them pay the value of the maximum between the $\left(Q_{T}+1\right)$ th highest bid and $v^{\mathrm{R}}$.

A buyer arriving at time $t \in[0, T)$, based on his private value, his knowledge of the arrival rate $\lambda$ and the distribution function $F$, the initial capacity offered $Q_{0}$, the auction remaining time $T-t$, the reservation value $v^{\mathrm{R}}$, and the posted price $\hat{P}$, chooses either to bid or to buy at the fixed price channel in order to maximize his expected utility. This participation strategy can be characterized by a threshold function $H(v)$ such that a buyer with valuation $v$ enters the auction only if his arrival time exceeds $H(v)$. Pictorially, $H(v)$ divides buyers' type space (valuation, arrival time) into two regions -as shown in Figure 1- one corresponding to the posted-price buyers and the other associated to the auction bidders.

The firm's problem is to design a single auction channel in the first model (by setting a value for $T, Q_{0}$ and $v^{\mathrm{R}}$ ), and dual auction and list price channels in the second model (by setting also a value $\hat{P})$, in order to maximize expected revenue, which is also exponentially discounted over time.

\subsection{Discussion of the model}

On a theoretical level, our model is in many ways a variation of the classical, single period, private-value auction model, but with a stochastic arrival process embedded in it. The existence of the alternative list price channel also distinguishes our formulation from the standard monopolistic auctioneer setting. Indeed, from the consumer's perspective, the existence of this alternative market enforces the non-participation in the auction value to be different than zero: the customer can eventually get the item outside the auction and still obtain a positive utility. This departure from the standard mechanism design literature (e.g. used in the Revenue Equivalence Theorem of Myerson [26], Riley and Samuelson [29] and Maskin and Riley [24]) makes this approach not straightforward. 


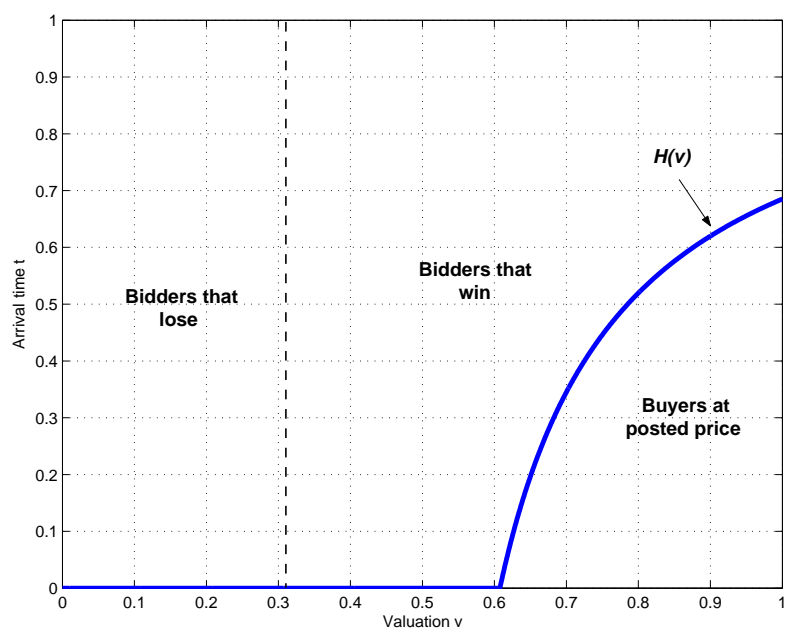

Figure 1: An example of a participation strategy $H(v)$ for the case $T=1$ and $v^{R}=0$. The vertical dashed line represents a possible realization of the auction price dividing bidders between winners and losers.

On a practical level, though our model captures important features of the real game, other characteristics are restrictive, and their implications are worth examining in greater detail. Rothkopf and Harstad [31] emphasize that typical assumptions in the auction literature (that are also shared by our model) like bidder symmetry and common knowledge of private valuation distributions, may not be verified in practice. Pinker et al. [28] point out that these assumptions are even more dubious for online auctions, where experts and novices participate in the same game. Similar to Etzion et al. [12], we add here an extra limitation of game theoretic models for online auctions: the common knowledge of the arrival rate of consumers. One might think that the seller has an advantage in this sense, since she can build statistics and learn more than the bidders through repeated runs of similar games (although in this paper, we analyze a single period problem).

Another property related to the arrival process in our model is the constant rate. Roth and Ockenfels [30] verify the surge in bids close to the end of auctions with sharp deadlines, the case we are looking at. ${ }^{2}$ They observe that when the auction end is not strict (i.e. it extends while there is bidding activity), peaks in the bidding activity are less significant. Though we think that our analysis could be extended to the variable arrival rate of customers, we have not explored this direction in detail.

We also assume risk neutrality of all players along the paper. That a large seller is risk neutral is quite reasonable, as typically each auction or dual channel outcome is a small proportion of her wealth, and she could be running repeated games over time. The fact that she is maximizing expected revenues seems quite natural then. Risk neutrality of bidders facing both auctions and list prices

\footnotetext{
${ }^{2}$ However, most of the last-minute bidding activity is originated in the proxy bidding devices of people that have previously arrived.
} 
is admittedly more arguable, but we assume that they base their choice on maximizing their own expected surplus.

The fact that when buyers arrive at the website they are just informed of the initial number of units $Q_{0}$ is plausible in the single auction channel; but it is certainly a limitation of the dual channel model. This contrasts with the business practice of posting buy now prices while running an auction (e.g. eBay.com): Buyers are informed of the number of remaining units when visiting the website. However, including this factor in the consumer choice behavior would make the analysis even more complex than what it is now, adding an extra dimension to the current space (valuation, time) for the participation decision. Nevertheless, we think that the kind of arguments and technical tools used in this paper can be applied to this extension as well.

We model the auction as a multiunit second-price sealed-bid auction. In standard single period auction theory, this auction format turns out to be strategically equivalent to the English auction ${ }^{3}$ (e.g. see Krishna [19]). Actually, the English auction is by far the most common online auction format (see Pinker et al. [28]), probably due to the simplicity of the optimal participation strategy. Some empirical research supports the theoretical equivalence between sealed-bid second-price and open English auctions in the online practice (e.g. Lucking-Reiley [21]).

Summarizing, our model is well supported on many practical features, but shares some limitations of the auction related literature. However, we introduce some important departures, mainly related to the analysis and design of simultaneous online and list price channels, that shed some light on the tradeoffs involved in the game among customers themselves, and between customers and the seller.

\section{Single auction channel}

We consider now the problem of a seller that uses exclusively the auction to sell the initial capacity $Q_{0}$. The distinguishing feature of our formulation is given by the fact that potential bidders arrive at different moments in time and their utilities are sensitive to delay. In particular, the arrival process is Poisson with rate $\lambda$, and the effective number of bidders at time $T$ has yet to be determined.

In our setting where the sealed $\left(Q_{T}+1\right)$-price auction ${ }^{4}$ operates in parallel to an infinite supply fixed price market, it is a dominant strategy for bidders with valuations below the posted price to bid their true values, and it is dominant for bidders above the posted price to bid the posted price. In other words, if a buyer with valuation $v$ decides to enter the auction then he will bid $b(v)$, where

$$
b(v)=\left\{\begin{array}{ll}
v & \text { if } v<\hat{P} \\
\hat{P} & \text { if } v \geq \hat{P}
\end{array}=\min \{v, \hat{P}\} .\right.
$$

\footnotetext{
${ }^{3}$ In a multiunit English auction, bids are open to all to see. As in the multiunit second-price sealed-bid auction, if $Q$ units are auctioned, the $Q$ highest bidders win, and all the winners pay the highest losing bid.

${ }^{4}$ In this single channel auction, $Q_{T}=Q_{0}$, since there are no items sold during the time window $[0, T)$.
} 
The optimality of $b(v)=v$ for a low-valuation bidder with $v<\hat{P}$ follows from noticing that for this buyer the auction is the only profitable channel from which he can get the object. In addition, it is well known that for the $\left(Q_{T}+1\right)$-price auction mechanism bidding the true valuation is a dominant strategy, i.e., the strategy $b(v)=v$ maximizes the low-valuation bidder's expected utility independently of other bidders' strategies. For high-valuation buyers with $v \geq \hat{P}$ the posted price channel is also an alternative to consider. Furthermore, and contrary to the auction channel, the fixed price channel has unlimited capacity and buyers with $v \geq \hat{P}$ know that they can always get a unit at a the fixed price $\hat{P}$. So, these high-valuation buyers will never bid above the posted price, that is, $b(v) \leq \hat{P}$. In addition, a bidding strategy with $b(v)<\hat{P}$ is also suboptimal because under the $\left(Q_{T}+1\right)$-price auction mechanism this strategy reduces the bidder probability of winning the auction (with respect to the strategy $b(v)=\hat{P}$ ) and at the same time does not affect the auction price in the case the bidder actually wins the auction. Thus, the high-valuation bidder is better off choosing $b(v)=\hat{P}$.

Interestingly, if we compare the bidding strategy $b(v)=\min \{v, \hat{P}\}$ of this model and the traditional bidding strategy $b(v)=v$ of the standard $\left(Q_{T}+1\right)$-price auction, we conclude that from the auctioneer's point of view the presence of an uncapacitated fixed price channel is equivalent to collapsing the range of valuations $[\hat{P}, \bar{v}]$ into a single value $\hat{P}$. For further details about the optimality of $b(v)=\min \{v, \hat{P}\}$, we refer the reader to Etzion et al. [12, Lemma 1].

Equation (2) characterizes the dominant bidding strategy for all bidders. The probability distribution of the number of bidders and their valuations is the only piece of information that we need to fully characterize the output of the auction. We address this issue in the first three subsections, where we concentrate exclusively on the buyers' optimal participation strategy for a fixed reservation price value $v^{\mathrm{R}}$. In Section 3.4, we consider the auctioneer's problem which optimizes over $v^{\mathrm{R}}$. Since $v^{\mathrm{R}}$ remains constant for most of this section, we find convenient to set it equal to zero $\left(v^{\mathrm{R}}=0\right)$ and to re-scale bidder's valuations and its probability distribution accordingly. That is,

$$
v \leftarrow \frac{v-v^{\mathrm{R}}}{1-v^{\mathrm{R}}}, \quad \hat{P} \leftarrow \frac{\hat{P}-v^{\mathrm{R}}}{1-v^{\mathrm{R}}}, \quad F(v) \leftarrow \frac{F(v)-F\left(v^{\mathrm{R}}+v\left(1-v^{\mathrm{R}}\right)\right)}{1-F\left(v^{\mathrm{R}}\right)}, \quad \lambda \leftarrow \lambda\left(1-F\left(v^{\mathrm{R}}\right)\right) .
$$

This transformation models two objectives. First, only those buyers with valuation greater than or equal to the auction reservation price are considered, the others are "discarded". This is without loss of generality since discarded buyers have no real impact on the auction output. The second objective is that under this scaling the range of valuations of the (non-discarded) buyers remains [0,1]. The

corresponding scaling of the posted price $\hat{P}$, distribution $F$, and arrival rate $\lambda$ follows from these two conditions.

\subsection{Participation strategy and auction price probability distribution}

Since information about the number of units $Q_{0}$ to auction as well as the probability distribution of customer valuations $F$ are common knowledge, we can characterize the decision of a buyer arriving at 
time $t$ with private valuation $v$ by a threshold function $H(v)$ such that the buyer will place a bid if and only if $t \geq H(v)$. The fact that we can represent the participation strategy for all $v$-buyers (i.e. , those buyers with valuation $v$ ) by a single threshold $H(v)$ is a consequence of the monotonicity of the utility function on the waiting time. In other words, if it is optimal for a $v$-buyer arriving at time $t$ to wait $(T-t)$ time units for the auction then it is also optimal for any other $v$-buyer arriving after $t$.

Two assumptions are used in this representation of the participation strategy. First, note that this characterization is based on the notion of a symmetric equilibrium in which all buyers use the same bidding function $H(v)$. In addition, we are also assuming that a buyer arriving at time $t$ is incapable to observe the number of bids already in the system. That is, we are assuming that the only information that buyers use to decide whether or not to enter the auction -besides $\lambda, T, Q_{0}$, and $F$ - is his arrival time and private valuation.

We will denote by $\mathcal{H}$ the set of participation thresholds from which buyers choose their strategies. In order to keep our formulation reasonably simple, avoiding measure theoretical technicalities, we assume that $\mathcal{H} \subseteq \mathcal{C}$, the set of continuous integrable functions. We will see that this apparently restrictive assumption is not fully needed since in equilibrium, participation strategies are always continuous. Note that by our definition, the elements of $\mathcal{H}$ are functions taking values in $[0, T]$. Furthermore, for any $H \in \mathcal{H}$ and any valuation $v \in[0, \hat{P}]$, we must have $H(v)=0$ and $b(v)=v$. This reflects the fact that any buyer with valuation in this range cannot afford to buy the product in the external market: the auction is his only potentially profitable channel, no matter his arrival time. In summary, we define the set of potential bidding strategies as the set of functions

$$
\mathcal{H}=\{H \in \mathcal{C}, H:[0,1] \rightarrow[0, T], \text { such that } H(v)=0 \text { for all } v \in[0, \hat{P}]\} .
$$

We still need to characterize what the equilibrium $H(v) \in \mathcal{H}$ looks like for $v \in(\hat{P}, 1]$. Buyers in this range will participate in the auction (and bid $b(v)=\hat{P}$ ) only if the expected auction price is small enough to compensate the disutility associated to waiting for the auction.

For any $H \in \mathcal{H}$, let us define two important random variables.

- $P_{H}$ : the auction price if all buyers use the participation strategy $H \in \mathcal{H}$.

- $P_{H}(v)$ : the auction price given that $(i)$ there is a $v$-buyer that has joined the auction, and $(i i)$ all other buyers use the participation strategy $H \in \mathcal{H}$.

In order to compute the probability distribution of these prices we need to estimate the number of bidders and their corresponding valuations. For this, consider $H \in \mathcal{H}$ and let us define for all $v \in \mathcal{V}$

$$
\Lambda_{H}(v) \triangleq \lambda \int_{v}^{1}(T-H(x)) \mathrm{d} F(x) \triangleq \lambda T \eta_{H}(v)
$$

where

$$
\eta_{H}(v) \triangleq \int_{v}^{1}\left(1-\frac{H(x)}{T}\right) \mathrm{d} F(x) .
$$


By the definition of the bidding function $H(v)$, the function $\Lambda_{H}(v)$ represents the average number of bidders with valuation in $[v, 1]$. Similarly, $\eta_{H}(v)$ represents the average fraction of arrivals with valuation in this range.

Let us denote by $B\left(\Lambda_{H}(v)\right)$ the random number of bidders with valuation greater than or equal to $v$. Since buyers arrive according to a Poisson process with rate $\lambda, B\left(\Lambda_{H}(v)\right)$ has a Poisson distribution with mean $\Lambda_{H}(v)$. Based on our assumptions that the elements in $\mathcal{H}$ are integrable and that $F(v)$ admits a density $f(v)$ in $\mathcal{V}$, we have that the functions $\Lambda_{H}(v)$ and $\eta_{H}(v)$ are well defined and continuous in $\mathcal{V}$.

We can now compute the probability distribution of $P_{H}$ and $P_{H}(v)$ given a symmetric participation strategy $H \in \mathcal{H}$. Under a $\left(Q_{0}+1\right)$-price auction, $P_{H}<x$ if and only if $(i) x>\hat{P}$, or $(i i)$ the number of bidders with valuation greater than or equal to $x$ is less than or equal to the number of objects in the auction. That is,

$$
P_{H}<x \quad \Longleftrightarrow \quad\{x>\hat{P}\} \vee\left\{B\left(\Lambda_{H}(x)\right) \leq Q_{0}\right\}
$$

Therefore, the probability distribution of $P_{H}$ is given by

$$
\mathbb{P}\left(P_{H}<x\right)=\left\{\begin{array}{cl}
1 & \text { if } x>\hat{P} \\
\mathbb{P}\left(B\left(\Lambda_{H}(x)\right) \leq Q_{0}\right)=\sum_{k=0}^{Q_{0}} \frac{\left(\Lambda_{H}(x)\right)^{k} \exp \left(-\Lambda_{H}(x)\right)}{k !} & \text { if } x \leq \hat{P}
\end{array}\right.
$$

Using a similar argument, it follows that

$$
\mathbb{P}\left(P_{H}(v)<x\right)=\left\{\begin{array}{cc}
1 & \text { if } x>\hat{P} \\
\mathbb{P}\left(B\left(\Lambda_{H}(x)\right)+\mathbb{1}(x \leq v) \leq Q_{0}\right)=\sum_{k=0}^{Q_{0}-\mathbb{1}(x \leq v) \frac{\left(\Lambda_{H}(x)\right)^{k} \exp \left(-\Lambda_{H}(x)\right)}{k !}} & \text { if } x \leq \hat{P}
\end{array}\right.
$$

where $\mathbb{1}(E)$ is the indicator function of event $E$. It also follows from the continuity of $\Lambda_{H}(x)$ that $\mathbb{P}\left(P_{H}<x\right)$ is continuous in $x \in[0,1]-\{\hat{P}\}$, and that $\mathbb{P}\left(P_{H}(v)<x\right)$ is continuous in $x \in[0,1]-\{\hat{P}, v \wedge$ $\hat{P}\}$.

\subsection{Characterization of a symmetric participation equilibrium $H(v)$}

In order to characterize a symmetric participation equilibrium (SPE) $H \in \mathcal{H}$, we use the following twostep approach. First, we look at a buyer's best-response participation strategy assuming that other buyers use a fixed strategy $H \in \mathcal{H}$. We will denote by $\mathcal{R}(H) \in \mathcal{H}$ this best-response participation strategy and refer to $\mathcal{R}$ as the best-response mapping on $\mathcal{H}$. Then, we impose the equilibrium condition $\mathcal{R}\left(H^{*}\right)=H^{*}$ to characterize the symmetric equilibrium $H^{*} \in \mathcal{H}$. Before moving into this analysis, we recall that the optimal strategy for buyers with valuation $v \leq \hat{P}$ (independent of $H$ ) is to enter the auction and so we must have $\mathcal{R}(H)(v)=0$ for all $v \in[0, \hat{P}]$.

Suppose a buyer -that we refer as buyer $\tau$ - arrives at time $\tau$ with private valuation $v_{\tau}>\hat{P}$ and suppose that every other buyer is using the participation strategy $H$. If buyer $\tau$ decides not to bid and 
buy a unit through the external fixed price channel, then his expected utility would be $u\left(\tau, \tau, v_{\tau}-\hat{P}\right)$. On the other hand, if he decides to bid then his profit would be $u\left(\tau, T, v_{\tau}-\hat{P}\right)$ if he does not get the object (since he can always buy the product in the external market at time $T$ ), and $u\left(\tau, T, v_{\tau}-P_{H}\left(v_{\tau}\right)\right.$ ) if he indeed gets the object. Thus, a rational buyer $\tau$ enters the auction only if

$$
u\left(\tau, T, v_{\tau}-\hat{P}\right)\left(1-\mathbb{P}\left(P_{H}\left(v_{\tau}\right)<\hat{P}\right)\right)+\mathrm{E}\left[u\left(\tau, T, v_{\tau}-P_{H}\left(v_{\tau}\right)\right) \mid P_{H}\left(v_{\tau}\right)<\hat{P}\right] \mathbb{P}\left(P_{H}\left(v_{\tau}\right)<\hat{P}\right) \geq u\left(\tau, \tau, v_{\tau}-\hat{P}\right),
$$

where $\mathbb{P}\left(P_{H}\left(v_{\tau}\right)<\hat{P}\right)$ is the probability that bidder $\tau$ gets one of the auctioned objects at a price strictly less than the posted price. We should still explicitly characterize this participation constraint in terms of the function $H(\cdot)$, but at this stage note that $H(\cdot)$ is embedded in the r.v. $P_{H}\left(v_{\tau}\right)$ (see equation (7)).

Without loss of generality, we will assume that $\hat{P}>0$ (otherwise, the auction is meaningless, since buyers will go directly to the external fixed price channel and get a unit at no risk). This is also consistent with the scaling in (3) where we set $v^{\mathrm{R}}=0$, since all the bidders with valuations smaller than $v^{\mathrm{R}}$ will have zero utility, and will quit without purchasing in any of the markets.

We compute the best-response strategy for buyer $\tau(\mathcal{R}(H))$ by looking at the threshold function that is consistent with (8). First, note that in our setting, where buyers have the exponentially discounted utility function defined in equation (1), condition (8) is equivalent to

$$
\frac{\hat{P}-\mathrm{E}\left[P_{H}\left(v_{\tau}\right) \mid P_{H}\left(v_{\tau}\right)<\hat{P}\right]}{v_{\tau}-\hat{P}} \mathbb{P}\left(P_{H}\left(v_{\tau}\right)<\hat{P}\right) \geq \exp (w(T-\tau))-1 .
$$

From this condition we conclude that in equilibrium the following inequality must be satisfied by the bidders:

$$
\mathrm{E}\left[P_{H}\left(v_{\tau}\right) \mid P_{H}\left(v_{\tau}\right)<\hat{P}\right]<\hat{P}
$$

That is, no buyer would have an incentive to bid if the expected auction price in case of wining is at least what he would pay in the external market, at no risk. The following proposition characterizes $\mathcal{R}(H)$ :

Proposition 1 For the exponential utility function (1), condition (9) is equivalent to

$$
\tau \geq T-\frac{1}{w} \ln \left(1+\int_{0}^{\hat{P}} \frac{\mathbb{P}\left(P_{H}\left(v_{\tau}\right)<x\right)}{v_{\tau}-\hat{P}} d x\right) .
$$

Thus, a buyer arriving at time $\tau$ with valuation $v_{\tau}$ enters the auction if and only if $\tau \geq \mathcal{R}(H)\left(v_{\tau}\right)$, where

$$
\mathcal{R}(H)\left(v_{\tau}\right) \triangleq\left\{\begin{array}{cl}
0 & \text { if } \quad v_{\tau} \in[0, \hat{P}] \\
{\left[T-\frac{1}{w} \ln \left(1+\int_{0}^{\hat{P}} \frac{\mathbb{P}\left(P_{H}\left(v_{\tau}\right)<x\right)}{v_{\tau}-\hat{P}} d x\right)\right]^{+}} & \text {if } \quad v_{\tau} \in(\hat{P}, 1] .
\end{array}\right.
$$


A couple of observations can be derived from this result. First, note that $\mathcal{R}(H)\left(v_{\tau}\right)$ is continuous in $v_{\tau} \in[0, \hat{P}]$ as well as continuous in $v_{\tau} \in(\hat{P}, 1]^{5}$. In addition, as $v_{\tau} \downarrow \hat{P}$ we have that $\mathcal{R}(H)\left(v_{\tau}\right) \rightarrow$ $0=\mathcal{R}(H)(\hat{P})$. This observation follows by noticing that

$$
\text { for all } H \in \mathcal{H}, \quad \lim _{v_{\tau} \downarrow \hat{P}} \int_{0}^{\hat{P}} \frac{\mathbb{P}\left(P_{H}\left(v_{\tau}\right)<x\right)}{v_{\tau}-\hat{P}} \mathrm{~d} x=+\infty,
$$

since the numerator of the integrand is strictly positive and bounded away from zero. Therefore, for any $H \in \mathcal{H}$ we have that the best-response strategy $\mathcal{R}(H)(v)$ is continuous in $[0,1]$ and so $\mathcal{R}$ effectively maps $\mathcal{H}$ into $\mathcal{H}$. Furthermore, since a SPE is characterized by the fixed-point condition $\mathcal{R}(H)=H$, we conclude that a symmetric participation equilibrium of this game is in fact continuous. Our next result extends this conclusion and shows that the best-response strategies are $K$-Lipschitz continuous $^{6}$ functions in $[0,1]$, for an appropriate constant $K>0$. This additional property of the bidding strategies becomes relevant in our proof of existence of an equilibrium. However, before we formally address this issue some extra notation and analysis are required.

We have already argued that any $\operatorname{SPE} H(v)$ must satisfy $H(v)=0$ in the range $v \in[0, \hat{P}]$. Based on Proposition 1, we can extend this range even more. In fact, note that $\mathcal{R}(H)(v)>0$ if

$$
1+\int_{0}^{\hat{P}} \frac{\mathbb{P}\left(P_{H}\left(v_{\tau}\right)<x\right)}{v_{\tau}-\hat{P}} \mathrm{~d} x<\exp (w T) .
$$

Since the left-hand side goes to infinite as $v_{\tau} \downarrow \hat{P}$, we can unambiguously define for every $H \in \mathcal{H}$

$$
v_{H} \triangleq 1 \wedge \underset{v \geq \hat{P}}{\operatorname{argmin}}\left\{1+\int_{0}^{\hat{P}} \frac{\mathbb{P}\left(P_{H}(v)<x\right)}{v-\hat{P}} \mathrm{~d} x \leq \exp (w T)\right\} .
$$

Note that by construction, for any $H \in \mathcal{H}$ we must have $\mathcal{R}(H)(v)=0$, for all $v \in\left[0, v_{H}\right]$. To obtain a lower bound on $v_{H}$ independent of $H$, we can solve

$$
\tilde{v} \triangleq \inf _{H \in \mathcal{H}}\left\{v_{H}\right\}
$$

Unfortunately, this is not a straightforward optimization problem for which we can compute the optimal solution $\tilde{v}$. However, we can obtain a lower bound on $\tilde{v}$ by mean of the following inequality.

$$
\text { For all } H \in \mathcal{H}, \quad 1+\int_{0}^{\hat{P}} \frac{\mathbb{P}\left(P_{H}(v)<x\right)}{v-\hat{P}} \mathrm{~d} x \geq 1+\int_{0}^{\hat{P}} \frac{\mathbb{P}\left(P_{0}(v)<x\right)}{v-\hat{P}} \mathrm{~d} x,
$$

where $P_{0}(v)$ stands for the auction price when $H=0$. Since for $x<\hat{P}, \mathbb{P}\left(P_{0}(v)<x\right)=\mathbb{P}\left(B\left(\Lambda_{0}(x)\right) \leq\right.$ $\left.Q_{0}-1\right)=\mathbb{P}\left(B(\lambda T[1-F(x)]) \leq Q_{0}-1\right)$, we get a lower bound on $\tilde{v}$ solving for $\underline{v}$ in

$$
1+\int_{0}^{\hat{P}} \frac{\mathbb{P}\left(B(\lambda T[1-F(x)]) \leq Q_{0}-1\right)}{\underline{v}-\hat{P}} \mathrm{~d} x=\exp (w T)
$$

\footnotetext{
${ }^{5}$ This follows from the continuity of $\mathbb{P}\left(P_{H}\left(v_{\tau}\right)<x\right)$ for $x \in[0, \hat{P}]$ (see equation $(7)$ ).

${ }^{6}$ We say that a real function $f(x)$ is $K$-Lipschitz continuous in $\mathcal{A}$ if for all $x, y \in \mathcal{A},|f(x)-f(y)| \leq K|x-y|$.
} 
or equivalently,

$$
\underline{v}=\hat{P}+\exp (-w T)\left(1+\int_{0}^{\hat{P}} \mathbb{P}\left(B(\lambda T[1-F(x)]) \leq Q_{0}-1\right) \mathrm{d} x\right) .
$$

It is possible that for some values of the problem's parameters (e.g. when $w T$ is small) such a $\underline{v}$ does not exist in $(\hat{P}, 1]$. In these cases we set $\underline{v}=\tilde{v}=1$. Note that if $\tilde{v}=1$ then for any $H \in \mathcal{H}$ we must have $\mathcal{R}(H)(v)=0$ for all $v \in(\hat{P}, 1]$ and so $H(v)=0$ is the unique SPE. We summarize our previous discussion in the following proposition.

Proposition 2 For the exponential utility function (1) and for all $H \in \mathcal{H}$, there is a positive constant $K$ (independent of $H$ ) such that the best-response strategy $\mathcal{R}(H)(v)$ is a $K$-Lipschitz continuous function that satisfies $\mathcal{R}(H)(v)=0$ for all $v \in[0, \tilde{v}]$. In addition, if $\tilde{v}=1$ then it is optimal for every buyer, independent of his arrival time and private valuation, to enter the auction. That is, if $\tilde{v}=1$ then $\{H(v)=0$ for all $v \in[0,1]\}$ is the unique (symmetric) participation strategy equilibrium.

Proposition 2 characterizes a SPE for those special cases in which every buyer enters the auction. For the general case, finding a symmetric equilibrium $H(v)$ or even proving its existence are not easy tasks. A standard way to approach the existence problem is to prove that the set of bidding strategies $\mathcal{H}$ has the fixed-point property (see Cheney [9, Section 7.1] for details) and that the bestresponse mapping $\mathcal{R}$ is continuous in $\mathcal{H}$. We will take this approach here, though we need to slightly modified our set of strategies $\mathcal{H}$ first. From our previous discussion and the result in Proposition 2, we can restrict the search of a symmetric equilibrium to those strategies $H$ in $\mathcal{H}$ that are $K$-Lipschitz continuous and satisfies $H(v)=0$ in $[0, \tilde{v}]$. For this reason, we redefine $\mathcal{H}$ to be this set:

$$
\mathcal{H} \triangleq\{H:[0,1] \rightarrow[0, T] \text { s.t. } H \text { is } K \text {-Lipschitz continuous and } H(v)=0 \text { in } v \in[0, \tilde{v}]\} \text {. }
$$

Note that by Proposition $2, \mathcal{R}$ is a well defined mapping from $\mathcal{H}$ to $\mathcal{H}$.

Theorem 1 The set of strategies $\mathcal{H}$ equipped with the uniform norm $\|X\|=\sup _{0 \leq v \leq 1}\{|X(v)|\}$ in $[0,1]$ exhibits the fixed-point property. In addition, for all $H, \tilde{H} \in \mathcal{H}$, the mapping $\mathcal{R}$ satisfies:

$$
\|\mathcal{R}(H)-\mathcal{R}(\tilde{H})\| \leq \frac{\lambda \beta\left(Q_{0}-1\right)}{w}\left(\int_{0}^{\hat{P}} \frac{(1-F(x))}{\tilde{v}-\hat{P}} d x\right)\|H-\tilde{H}\| .
$$

Therefore, $\mathcal{R}$ is a continuous mapping and there always exists a SPE. In addition, if

$$
\frac{\lambda \beta\left(Q_{0}-1\right)}{w}\left(\int_{0}^{\hat{P}} \frac{(1-F(x))}{\tilde{v}-\hat{P}} d x\right)<1
$$

then $\mathcal{R}$ is a contraction. In this case, the fixed point $\mathcal{R}\left(H^{*}\right)=H^{*}$ is guaranteed to be unique and can be found through the iteration $H^{n+1}=\mathcal{R}\left(H^{n}\right)$ starting at an arbitrary $H^{1} \in \mathcal{H}$.

We conclude our characterization of a SPE with the following property. 
Proposition 3 For the exponential utility function (1), a SPE $H^{*}(v)$ is an increasing and concave function of $v \in\left[v_{H^{*}}, 1\right]$.

The result implies that in equilibrium buyers with large private valuation are less likely to participate in the auction.

To summarize, in this section we have characterized buyers' best-response participation strategy through a continuous mapping $\mathcal{R}$ and we have shown that there always exists a $\operatorname{SPE} H^{*}(v)$ satisfying $H^{*}=\mathcal{R}\left(H^{*}\right)$ which is identically zero in then range $\left[0, v_{H^{*}}\right]$ and $K$-Lipschitz continuous, increasing, and concave in the range $\left[v_{H^{*}}, 1\right]$. Moreover, we have been able to characterize those cases in which either $H^{*}(v)=0$ or the algorithm $H^{n+1}=\mathcal{R}\left(H^{n}\right)$ effectively converges to $H^{*}$. Unfortunately, this analysis is not exhaustive in the sense that there are instances of the problem for which we do not have a guaranteed method for computing the equilibrium. ${ }^{7}$ For this reason, in the next section we consider an asymptotic regime for which explicit solutions are obtained. As we will see, numerical experiments reveal that the behavior of the asymptotic approximation is satisfactorily accurate.

Other important feature to point out before proceeding is the existence of the value $v_{H^{*}}$ defined in (13). This means that in equilibrium, all customers with valuations slightly above the posted price (i.e. with valuation $v \in\left[\hat{P}, v_{H^{*}}\right]$ ) will join the auction regardless the arrival time.

\subsection{Asymptotic analysis}

In this section we characterize the outcome of the auction using asymptotic analysis. In particular, we consider the limiting case in which both the number of units $Q_{0}$ and the average arrival rate $\lambda$ grow proportionally large. In this regime, we show that characterizing the bidders' strategy (i.e., the threshold function $H(v)$ ) is equivalent to solving a deterministic problem which we can do efficiently (Theorem 3).

Consider a sequence of instances of the problem indexed by $n$ and let $Q_{0}^{n}$ and $\lambda^{n}$ be the corresponding number of units to auction and demand rate for instance $n$, respectively. All other parameters are kept independent of $n$. The asymptotic regime that we consider is defined by:

$$
\lim _{n \rightarrow \infty} \frac{Q_{0}^{n}}{n}=Q_{0} \quad \text { and } \quad \lim _{n \rightarrow \infty} \frac{\lambda^{n}}{n}=\lambda .
$$

For each instance $n$ of the problem, we let $\rho^{n} \triangleq \frac{Q_{0}^{n}}{\lambda^{n} T}$. Then,

$$
\lim _{n \rightarrow \infty} \rho^{n}=\rho, \text { for } \rho=\frac{Q_{0}}{\lambda T}
$$

\footnotetext{
${ }^{7}$ In practice, we have been able to find a SPE for all instances that we have tested using the following small step size version of the iteration in Theorem 1 (see Bertsekas and Tsitsiklis [3, Chapter 4]):

$$
H^{n+1}=\alpha H^{n}+(1-\alpha) \mathcal{R}\left(H^{n}\right),
$$

where $\alpha \in[0,1)$ is empirically selected.
} 
For future references, we refer to $\rho$ as the excess supply ratio which can be viewed as a proxy for the average number of units available per arriving customer. In this respect, the case $\rho<1$ reflects the most interesting situation in which, on average, there are less units than customers.

The auction price for instance $n$ given that a $v$-buyer enters the auction is $P_{H}^{n}(v)$, with distribution:

$$
\mathbb{P}\left(P_{H}^{n}(v)<x\right)=\left\{\begin{array}{cl}
1 & \text { if } x>\hat{P} \\
\mathbb{P}\left(B\left(\Lambda_{H}^{n}(x)\right) \leq Q_{0}^{n}-\mathbb{1}(x \leq v)\right) & \text { if } x \leq \hat{P},
\end{array}\right.
$$

where $\Lambda_{H}^{n}(x)=\lambda^{n} T \eta_{H}(x)$.

We need the following preliminary result:

Lemma 1 Let $B_{i}\left(\mu_{n}\right)$ be a sequence of i.i.d Poisson random variable with mean $\mu_{n}$, and let $y_{n}$ be an increasing sequence of nonnegative integers. For each $n$, define $S_{y_{n}} \triangleq \sum_{i=1}^{y_{n}} B_{i}\left(\mu_{n}\right)$. Suppose that:

$$
\lim _{n \rightarrow \infty} y_{n}=\infty, \quad \text { and } \quad \lim _{n \rightarrow \infty} \mu_{n}=\mu
$$

Then, the moment generating function of the r.v. $Y_{n} \triangleq S_{y_{n}} / y_{n}$ converges to a constant $\exp (\theta \mu)$, and hence $Y_{n}$ converges weakly ${ }^{8}$ to the constant $\mu$.

We are now ready to characterize the asymptotic regime:

Theorem 2 Suppose the participation strategy $H(v)$ is fixed. Then, in the limit as $n \rightarrow \infty$, the auction price $P_{H}^{n}(v)$ converges weakly to the constant $P_{H}^{\infty}(v)=\min \left\{\hat{P}, \eta_{H}^{-1}(\rho)\right\}$, where $\eta_{H}^{-1}(\rho) \triangleq \min \{v \in[0,1]$ : $\left.\eta_{H}(v) \leq \rho\right\}$

It follows from Theorem 2 that the probability distribution of the limiting auction price is

$$
\mathbb{P}\left(P_{H}^{\infty}(v)<x\right)= \begin{cases}1 & \text { if } x>\hat{P} \\ 1 & \text { if } \rho>\eta_{H}(x) \text { and } x \leq \hat{P} \\ 0 & \text { if } \rho<\eta_{H}(x) \text { and } x \leq \hat{P}\end{cases}
$$

Suppose $x \leq \hat{P}$ and $\rho<\eta_{H}(x)$, that is, there are on average more bidders with valuation at least $x$ than units to auction, $Q_{0}<\Lambda_{H}(x)$. In this situation, there is scarcity of units for bidders with valuation greater than or equal to $x$. Note that the monotonicity of $\eta_{H}(x) \operatorname{implies} x \leq \min \left\{\hat{P}, \eta_{H}^{-1}(\rho)\right\}$ and it follows from Theorem 2 that the auction price will be higher than $x$. On the other hand, when there are more units to auction than bidders with valuation at least $x$, that is $\rho>\eta_{H}(x)$, the final auction price will be lower than $x$ with certainty.

\footnotetext{
${ }^{8} \mathrm{~A}$ sequence of distribution functions is said to converge weakly to a limit $F$ (written $\left.F_{n} \Rightarrow F\right)$ if $F_{n}(y) \rightarrow F(y$ ) for all $y$ that are continuity points of $F$. A sequence of random variables $X_{n}$ is said to converge weakly or converge in distribution to a limit $X_{\infty}$ (written $X_{n} \Rightarrow X_{\infty}$ ) if their distribution functions $F_{n}(x) \triangleq P\left(X_{n} \leq x\right)$ converge weakly. See Durrett [11, Section 2.2].
} 
Note that the limiting auction price $P_{H}^{\infty}(v)$ does not depend on $v$ since as the number of units and buyers goes to infinity, the auction price is unaffected by the decision of one particular bidder. In other words, we can think of this asymptotic regime as one in which we have a continuum of marginal buyers each one having no impact on the overall outcome of the auction. In this respect, the asymptotic regime under consideration is of the fluid type. For notational convenience, let us define $P_{H} \triangleq P_{H}^{\infty}(v)$.

Suppose buyer $\tau$ with valuation $v_{\tau}$ arrives at time $\tau$ and suppose that every other buyer is using the participation strategy $H$.

- If $v_{\tau} \in[0, \hat{P}]$ the buyer enters the auction independently of the auction price since the posted price exceeds his valuation. Thus, we have that $\mathcal{R}(H)\left(v_{\tau}\right)=0$ for all $v_{\tau} \in[0, \hat{P}]$.

- If $v_{\tau} \in(\hat{P}, 1]$ then it is optimal for buyer $\tau$ to participate in the auction only if

$$
\left(v_{\tau}-P_{H}\right) \exp (-w(T-\tau)) \geq v_{\tau}-\hat{P}, \text { or equivalently } \tau \geq h\left(v_{\tau}\right)
$$

for the auxiliary threshold function

$$
h\left(v_{\tau}\right)=T-\frac{1}{w} \ln \left(\frac{v_{\tau}-P_{H}}{v_{\tau}-\hat{P}}\right) .
$$

The logarithm above goes to $\infty$ as $v_{\tau} \downarrow \hat{P}$ and so buyers with valuation greater but close to $\hat{P}$ will enter the auction. In fact, let us define $v_{H}$ to be the unique positive root of the equation

$$
T-\frac{1}{w} \ln \left(\frac{v-P_{H}}{v-\hat{P}}\right)=0 \quad \text { which is equivalent to } v_{H} \triangleq \frac{\hat{P} \exp (w T)-P_{H}}{\exp (w T)-1} \geq \hat{P},
$$

where the inequality follows from the condition $P_{H} \leq \hat{P}$. Then, by condition (17) buyer $\tau$ with valuation $v_{\tau} \in\left(\hat{P}, v_{H}\right]$ will always enter the auction independently of his arrival time $\tau$. On the other hand, buyer $\tau$ with valuation $v_{\tau}$ greater than $v_{H}$ will enter the auction only if his arrival time is greater than $\mathcal{R}(H)\left(v_{\tau}\right)$, where

$$
\mathcal{R}(H)\left(v_{\tau}\right)=T-\frac{1}{w} \ln \left(\frac{v_{\tau}-P_{H}}{v_{\tau}-\hat{P}}\right), \quad v_{\tau} \in\left[v_{H}, 1\right] .
$$

We note that we have implicitly assumed that $v_{H} \leq 1$, however, depending on the values of $P_{H}$, $\hat{P}, w$, and $T$, it is possible that $v_{H}>1$. So, in order to avoid this technicality, we redefine $v_{H}$ as follows

$$
v_{H} \triangleq \min \left\{\frac{\hat{P} \exp (w T)-P_{H}}{\exp (w T)-1}, 1\right\}
$$

Clearly, if $v_{H}=1$ then every buyer will enter the auction independently of his arrival time, in which case $H^{*}(v)=0$ for all $v \in[0,1]$. 
In summary, given $H \in \mathcal{H}$ and the associated auction price $P_{H}$, the best-response participation strategy $\mathcal{R}(H)$ satisfies

$$
\mathcal{R}(H)(v)=\left\{\begin{array}{cl}
0 & \text { if } v \in\left[0, v_{H}\right] \\
T-\frac{1}{w} \ln \left(\frac{v-P_{H}}{v-\hat{P}}\right) & \text { if } v \in\left[v_{H}, 1\right]
\end{array}\right.
$$

For $v \in\left[v_{H}, 1\right]$, and given that $P_{H} \leq \hat{P}$,

$$
\frac{\mathrm{d}}{\mathrm{d} v} \mathcal{R}(H)(v)=-\frac{1}{w}\left(\frac{1}{v-P_{H}}-\frac{1}{v-\hat{P}}\right)>0 \text { and } \frac{\mathrm{d}}{\mathrm{d}^{2} v} \mathcal{R}(H)(v)=-\frac{1}{w}\left(-\frac{1}{\left(v-P_{H}\right)^{2}}+\frac{1}{(v-\hat{P})^{2}}\right)<0 .
$$

That is, $\mathcal{R}(H)(v)$ is increasing and concave in $v$, for all $v \geq v_{H}$.

In order to determine the equilibrium value of the auction price $P_{H^{*}}$ and the corresponding participation strategy $H^{*}(v)$, we have to impose the equilibrium condition $\mathcal{R}\left(H^{*}\right)=H^{*}$. In this asymptotic regime, we can solve this fixed-point condition efficiently using equation (19) and Theorem 2.

Theorem 3 In the asymptotic regime under consideration, the auction price $P_{H^{*}}$ is the unique solution in $[0, \hat{P}]$ to the equation

$$
F\left(v_{H^{*}}\right)-F\left(P_{H^{*}}\right)+\frac{1}{w T} \int_{v_{H^{*}}}^{1} \ln \left(\frac{v-P_{H^{*}}}{v-\hat{P}}\right) d F(v)=\min \left\{\rho, \eta_{H^{*}}(0)\right\},
$$

where $v_{H^{*}}=\min \left\{\frac{\hat{P} \exp (w T)-P_{H^{*}}}{\exp (w T)-1}, 1\right\}$ and the SPE strategy $H^{*}(v)$ is given by

$$
H^{*}(v)=\left\{\begin{array}{cl}
0 & \text { if } v \in\left[0, v_{H^{*}}\right] \\
T-\frac{1}{w} \ln \left(\frac{v-P_{H^{*}}}{v-\hat{P}}\right) & \text { if } v \in\left[v_{H^{*}}, 1\right]
\end{array}\right.
$$

The next result characterizes two extreme outputs of the game and follows directly from Theorem 3 :

Corollary 1 In the asymptotic regime, it follows that:

a) The auction price equals the reservation price, that is $P_{H^{*}}=0$, if

$$
F\left(v_{H^{*}}\right)+\frac{1}{w T} \int_{v_{H^{*}}}^{1} \ln \left(\frac{v}{v-\hat{P}}\right) d F(v) \leq \rho, \text { where } v_{H^{*}}=\min \left\{\frac{\hat{P} \exp (w T)}{\exp (w T)-1}, 1\right\} \text { and }
$$

b) All arriving buyers enter the auction, that is $H^{*}(v)=0$ for all $v \in[0,1]$, if

$$
F^{-1}(1-\rho) \leq 1-\exp (w T)(1-\hat{P}) \text { in which case } P_{H^{*}}=F^{-1}(1-\rho) .
$$


Asymptotic Approximation: Computational Experiments

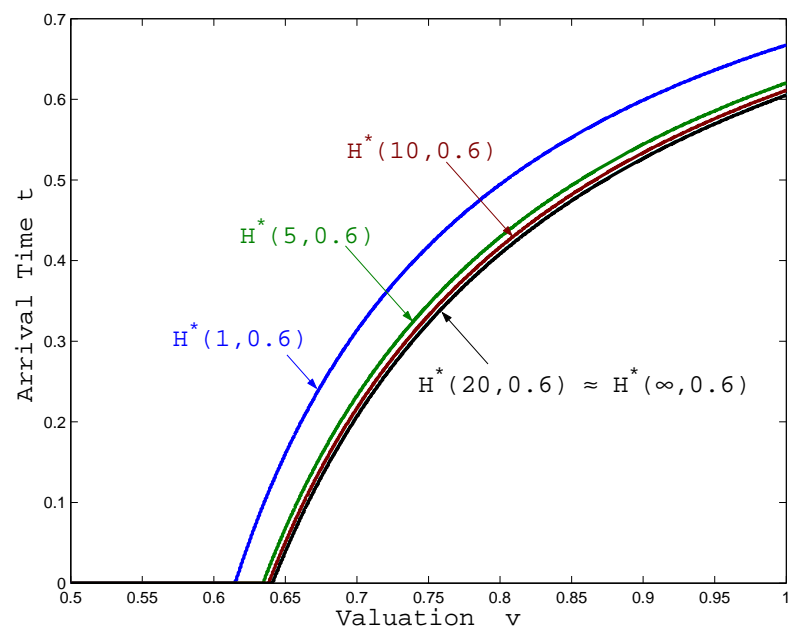

\begin{tabular}{||c|c|c|c||}
\hline \hline$Q_{0}$ & $P_{H}$ & $P_{H}^{\text {Approx }}$ & Error \\
\hline 1 & 0.1249 & 0.1346 & $7.20 \%$ \\
\hline 5 & 0.1930 & 0.1979 & $2.43 \%$ \\
\hline 10 & 0.2144 & 0.2168 & $1.12 \%$ \\
\hline 20 & 0.2312 & 0.2319 & $0.30 \%$ \\
\hline \hline
\end{tabular}

Figure 2: Asymptotic approximation for the case with valuations uniformly distributed in $[0,1]$, and $T=w=1$, $\hat{P}=0.5, \rho=0.6$. In this case, the asymptotic price is $P_{H^{*}}^{\infty}=0.2578$.

By introducing the notation $H^{*}\left(Q_{0}, \rho\right)$ when referring to the optimal participation strategy if the seller has $Q_{0}$ units to auction and the excess supply ratio $\left(Q_{0} / \lambda T\right)$ is equal to $\rho$, in Figure 2 we compare the optimal asymptotic participation strategy $H^{*}(\infty, 0.6)$ (computed using Theorem 3 ) to four optimal bidding strategies $H^{*}(1,0.6), H^{*}(5,0.6), H^{*}(10,0.6)$, and $H^{*}(20,0.6)$ (computed numerically using the iteration in Theorem 1). From the graph on the left of Figure 2, we can see that the asymptotic approximation mimics quite closely buyers' participation strategy even for small values of $Q_{0}$. As a matter of the fact, for values of $Q_{0}$ greater than 10 units, the bidding strategies $H^{*}\left(Q_{0}, 0.6\right)$ and $H^{*}(\infty, 0.6)$ are almost indistinguishable.

The table on the right of Figure 2 compares the expected price of the auction $P_{H}$ with the approximated value $P_{H}^{\text {Approx }}$ obtained using the asymptotic participation strategy. In other words, $P_{H}^{\text {Approx }}$ is the expected auction price if every buyer uses the participation strategy $H^{*}(\infty, \rho)$. As we can see, for a single-unit auction the error on the estimate is about $7 \%$. However, for moderate multi-unit auctions (with 5 or more items) the quality of the approximation improves considerably fast. With 20 items the approximation is almost exact. As we expect, both the auction price $P_{H}$ and the approximated auction price $P_{H}^{\text {Approx }}$ converge to the asymptotic price $P_{H}^{\infty}$ as $Q_{0}$ goes to infinity. The quality of results reported in this example were systematically replicated in all instances of the problem that we have considered.

We conclude this section, discussing our results on a particular instance of the problem with uniform valuations, a distribution widely considered in the auction literature.

\section{Example: Uniform Distribution Case.}

Suppose the valuations are uniformly distributed in $[0,1]$, that is, $F(v)=v$. In this case, we can apply Theorem 3 to get the following cases: 
- If $\hat{P} \leq \hat{P}_{1}(\rho, w T)$ where $\hat{P}_{1}(\rho, w T)$ solves

$$
\frac{-1}{w T}\left[\left(1-\hat{P}_{1}\right) \ln \left(1-\hat{P}_{1}\right)+\hat{P}_{1} \ln \left(\frac{\hat{P}_{1}}{\exp (w T)-1}\right)\right]=\min \{1, \rho\}
$$

then $P_{H^{*}}=0$ and $H^{*}(v)=\left[T-\frac{1}{w} \ln \left(\frac{v}{v-\hat{P}}\right)\right]^{+}$.

- If $\hat{P} \geq \hat{P}_{2}(\rho, w T) \triangleq 1-\exp (-w T)\left(1-(1-\rho)^{+}\right)$then $H^{*}=0$ and $P_{H^{*}}=(1-\rho)^{+}$.

- If $\hat{P}_{1}(\rho, w T)<\hat{P}<\hat{P}_{2}(\rho, w T)$ then the auction price $P_{H^{*}} \in(0, \hat{P})$ solves $\eta_{H^{*}}\left(P_{H^{*}}\right)=\rho$, which in this case is the same as

$$
\frac{1}{w T}\left[\left(1-P_{H^{*}}\right) \ln \left(1-P_{H^{*}}\right)-(1-\hat{P}) \ln (1-\hat{P})-\left(\hat{P}-P_{H^{*}}\right) \ln \left(\frac{\hat{P}-P_{H^{*}}}{\exp (w T)-1}\right)\right]=\rho \text {. }
$$

In words, $\hat{P}_{1}(\rho, w T)$ is a lower bound on the posted price over which there will be enough people in the auction such that the outcoming auction price will be positive. $\hat{P}_{2}(\rho, w T)$ is the minimum posted price such that all customers will participate in the auction. Figure 3 plots the auction price as a function of the posted price. As we can see, for low values of the posted

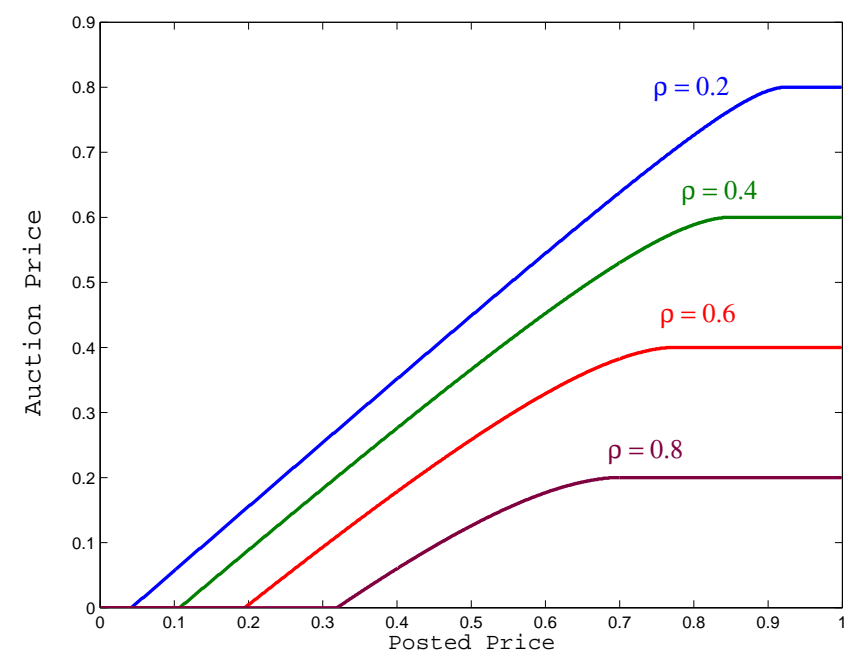

Figure 3: Auction price $P_{H}$ as a function of the posted price $\hat{P}$ for four different values of the excess supply ratio $\rho$. In this numerical example $w T=1$.

price $\hat{P}$, the auction price equals the reservation price zero. The intuition in this case is that a low posted price will induce a large fraction of the high-valuation buyers to purchase at the posted price and so there will be fewer bidders left on the auction; bidders that, moreover, have a low valuation. As we can see from Figure $3, \hat{P}_{1}(\rho, w T)$ increases with $\rho$ reflecting the fact that the higher the number of units in the auction the lower the value of the bid of the last winning bidder. On the other extreme, $\hat{P}_{2}(\rho, w T)$ decreases with $\rho$; that is, the higher the $\rho$, the lower the posted price needed to induce all the players to participate in the auction. In 
summary, the auction price $P_{H^{*}}$ is an nondecreasing function of $\hat{P}$ given by

$$
P_{H^{*}}=\mathcal{P}(\hat{P}, \rho, w T) \triangleq\left\{\begin{array}{cl}
0 & \text { if } \hat{P} \in\left[0, \hat{P}_{1}(\rho, w T)\right] \\
\text { solves } \eta_{H^{*}}\left(P_{H^{*}}\right)=\rho & \text { if } \hat{P} \in\left[\hat{P}_{1}(\rho, w T), \hat{P}_{2}(\rho, w T)\right] . \\
(1-\rho)^{+} & \text {if } \hat{P} \in\left[\hat{P}_{2}(\rho, w T), 1\right]
\end{array}\right.
$$

The corresponding number of units sold in the auction $Q_{H^{*}}$ satisfies

$Q_{H^{*}}=\lambda T \mathcal{Q}(\hat{P}, \rho, w T)$, where $\mathcal{Q}(\hat{P}, \rho, w T) \triangleq\left\{\begin{array}{cl}\eta_{H^{*}}(0) & \text { if } \hat{P} \in\left[0, \hat{P}_{1}(\rho, w T)\right] \\ \rho & \text { if } \hat{P} \in\left[\hat{P}_{1}(\rho, w T), \hat{P}_{2}(\rho, w T)\right] \\ \min \{1, \rho\} & \text { if } \hat{P} \in\left[\hat{P}_{2}(\rho, w T), 1\right],\end{array}\right.$

and

$$
\eta_{H^{*}}(0)=\left\{\begin{array}{cl}
\frac{1}{w T}\left[\hat{P} \ln \left(\frac{(1-\hat{P})(\exp (w T)-1)}{\hat{P}}\right)-\ln (1-\hat{P})\right] & \text { if } \hat{P} \exp (w T)<\exp (w T)-1 \\
1 & \text { if } \hat{P} \exp (w T) \geq \exp (w T)-1 .
\end{array}\right.
$$

\subsection{Auctioneer's optimization problem}

After having characterized the strategic behavior of the customers, in this section we analyze the seller's design of the auction channel with the objective of maximizing revenues. For simplicity, and provided the accuracy of the approximation shown in Section 3.3, we will work on the simple asymptotic regime, assuming that the original distribution of valuations is $F \triangleq \operatorname{Unif}[0,1]$, and that the seller's revenue is exponentially discounted over time. We denote by $\alpha>0$ this discount rate factor, which can differ from the $w$ shared by the customers.

The first step is to revert the scaling in (3) and formulate the auctioneer's problem in terms of the original reservation price $v^{\mathrm{R}}$. Specifically, we unscale the arrival rate, the supply-excess ratio, and the posted price as follows

$$
\lambda \rightarrow \lambda\left(1-v^{\mathrm{R}}\right), \quad \rho \rightarrow \frac{Q_{0}}{\lambda T\left(1-v^{\mathrm{R}}\right)}=\frac{\rho}{1-v^{\mathrm{R}}}, \quad \hat{P} \rightarrow \frac{\hat{P}-v^{\mathrm{R}}}{1-v^{\mathrm{R}}} .
$$

Based on this transformation, and the auction output for the uniform case given by (21) and (22), the unscaled auction price and number of units auctioned are given by

$$
P_{H^{*}}\left(v^{\mathrm{R}}, T\right)=v^{\mathrm{R}}+\left(1-v^{\mathrm{R}}\right) \mathcal{P}\left(\frac{\hat{P}-v^{\mathrm{R}}}{1-v^{\mathrm{R}}}, \frac{\rho}{1-v^{\mathrm{R}}}, w T\right) \text { and } Q_{H^{*}}\left(v^{\mathrm{R}}, T\right)=\lambda T\left(1-v^{\mathrm{R}}\right) \mathcal{Q}\left(\frac{\hat{P}-v^{\mathrm{R}}}{1-v^{\mathrm{R}}}, \frac{\rho}{1-v^{\mathrm{R}}}, w T\right),
$$

respectively. The first aspect we study is the selection of the optimal reservation price. Suppose that the seller holds $Q_{0}$ units of capacity, receives customers with an arrival rate $\lambda$, conducts an auction of length $T$, and faces a constant external posted price $\hat{P}$, independent of all other parameters of the problem. In this context, the seller has to solve (numerically) the following problem:

$$
V_{A}\left(Q_{0}, T\right)=\max _{0 \leq v^{\mathrm{R}} \leq 1}\left\{\exp (-\alpha T) P_{H^{*}}\left(v^{\mathrm{R}}, T\right) Q_{H^{*}}\left(v^{\mathrm{R}}, T\right)\right\}
$$


Figure 4 shows that there is an optimal (or range of optimal) reservation price $v^{\mathrm{R}}$. Specifically, when $\rho=Q_{0} /(\lambda T) \geq 1$, all the winners pay the reservation price, and the tradeoff is between the number of those bidders and the reservation price announced. The revenue function is unimodal in that case. When $\rho<1$, there is a range of reservation prices (starting at $v^{\mathrm{R}}=0$ ) such that all the $Q_{0}$ units are allocated to the winners (i.e. the flat part of the curve in case (b)). However, for a higher $v^{\mathrm{R}}$, the number of bidders is less than $Q_{0}$, and revenues start to decrease.

\section{Optimization of the Reservation Price for the Single Channel Case}

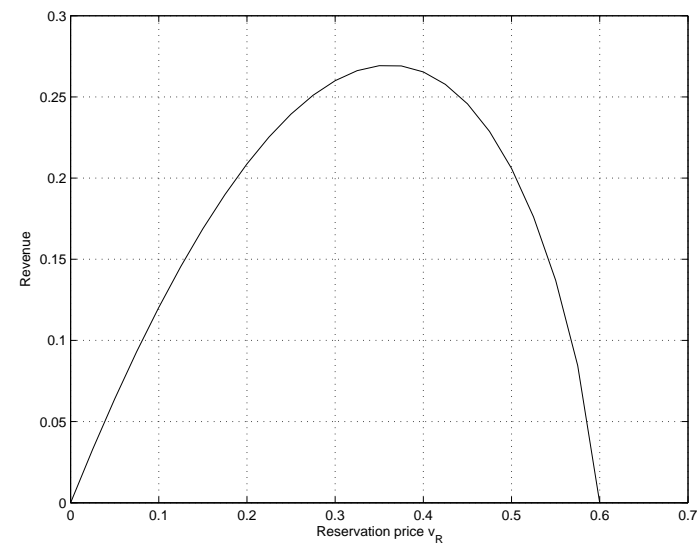

Case (a): $\rho \geq 1$

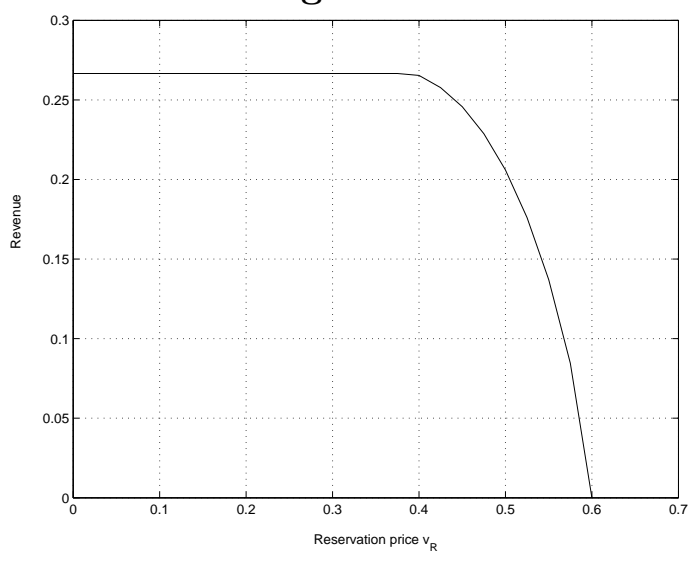

Case (b): $\rho<1$

Figure 4: Auctioneer's revenue as a function of the reservation price $v^{R}$, auction duration $T=1$, original valuations with distribution $F \triangleq \operatorname{Unif}[0,1], \lambda=10, w=1, \alpha=2$, and external posted price $\hat{P}=0.6$. In case (a), $Q_{0}=30$, and the optimum is $v^{R}=0.35$. In case (b), $Q_{0}=5$, and any $v^{R} \in[0,0.375]$ is optimal.

If the auctioneer wants to simultaneously optimize $T$ and $v^{\mathrm{R}}$ for a given capacity $Q_{0}$, then she must solve: $\max _{T \geq 0} V_{A}\left(Q_{0}, T\right)$. Figure 5 illustrates this for $Q_{0}=15$. The revenue function has a global maximum at $v^{\mathrm{R}}=0.45$ and $T=0.4$.

\section{Dual channel with static list price}

We consider here the case of a monopolistic seller who has $Q_{0}$ units to sell through two different channels: a list price channel, in which she sets a constant list price $\hat{P}$ that will be kept during the whole horizon of length $T$, and the auction that will take place at the end, with the remaining $Q_{T}$ units.

One of the important differences of this model with respect to the one discussed in the previous section is that the fixed price channel has now a limited capacity, bounded above by $Q_{0}$. Therefore, in this new setting the optimal bidding strategy for bidders is $b(v)=v$ as opposed to the strategy $b(v)=\min \{\hat{P}, v\}$ of the previous section. To see this, note that in this case a high-valuation buyer (i.e. with $v>\hat{P}$ ) that enters the auction and looses will not get the object at all, since all the units 


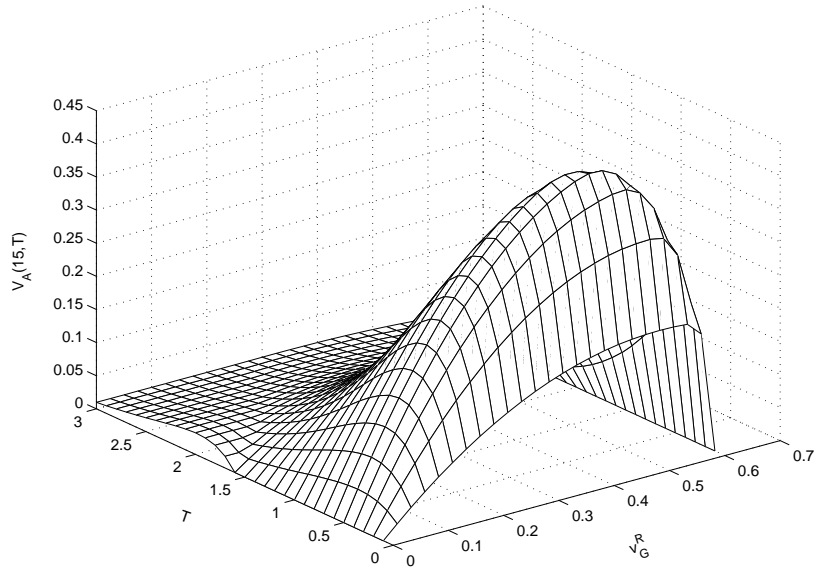

Figure 5: Revenue function for $Q_{0}=15$, original valuations with distribution $F \triangleq$ Unif $[0,1], \lambda=10, w=1, \alpha=2$, and external posted price $\hat{P}=0.6$.

are cleared at $T$ among buyers with valuations above $v^{\mathrm{R}}$. So, once this high-valuation buyer decides to enter the auction then the game he plays is exactly equivalent to a $\left(Q_{T}+1\right)$-price auction for which we know the optimal strategy is to bid the true valuation, $b(v)=v{ }^{9}$

Similar to the previous section, we analyze the buyers' participation problem under the scaling in (3), which we only revert at the end of this section when we study the seller's optimization problem. For simplicity, we will keep the same notation that we use in the single channel case with the understanding that the value of some quantities ( $\operatorname{such}$ as $\tilde{v}, v_{H}$, or $P_{H}$ ) will be slightly different.

\subsection{Characterization of a symmetric participation equilibrium $H(v)$}

Suppose $\hat{P}$ has been selected. Like in the previous section, we restrict buyers' participation strategies to the set

$$
\mathcal{H}=\{H \in \mathcal{C}:[0,1] \rightarrow[0, T] \text { such that } H(v)=0, \text { for all } v \in[0, \hat{P}]\},
$$

and we characterize the decision of a buyer by mean of a threshold function $H \in \mathcal{H}$, such that a buyer arriving at time $t$ with valuation $v_{t}$ will join the auction only if $H\left(v_{t}\right) \leq t$. The fact that $H\left(v_{t}\right)$ does not depend explicitly on the number of bids at time $t$ nor on $Q_{t}$ (number of unsold units at time $t$ ) means that the buyer is incapable to observe the number of bids already in the system, and the exact number of remaining units. That is, at any time $t \in[0, T]$, buyer $t$ only knows the initial quantity $Q_{0}$.

Similarly to Section 3.2, we analyze the buyer's problem at his arriving time in order to compute $\mathcal{R}(H)$, the best-response participation strategy given that all other buyers use the strategy $H$. As we

\footnotetext{
${ }^{9}$ Note that in this case $Q_{T}$ is not a fixed quantity but a random variable that depends on the initial number of units $Q_{0}$ and the number of buyers that select the fixed price channel during $[0, T)$. The strategy is dominant even for this case where the number of units to auction $Q_{T}$ is uncertain. The argument is similar to the one used for the standard second-price auction.
} 
will see, much of the analysis of Section 3.2 remains unchanged as long as we redefine $P_{H}(v)$ as follows:

$P_{H}(v)$ is the (random) auction price given that $(i)$ there is a $v$-buyer that has joined the auction, (ii) all other buyers use the participation strategy $H$, and (iii) the (random) number $Q_{T}$ of items left for the auction is equal to the difference between the initial value $Q_{0}$ and the number of items purchased through the fixed-price channel during $[0, T]$. We also use the convention $P_{H}(v)=1$ if $Q_{T}=0$.

As we will see shortly, an SPE in this dual channel case depends on the probability distribution of $P_{H}(v)$ and the value of $\mathbb{P}\left(Q_{\tau}>0 \mid H\right)$, the probability that at time $\tau$ there are still unsold units given that buyers use the participation strategy $H$. In order to compute these quantities, we follow an approach similar to the one developed for the single auction channel: We fix $H$, and in addition we define

$$
\begin{array}{ll}
\Lambda_{H+}(\tau) \triangleq \lambda T \eta_{H+}(\tau), \quad \text { for } \quad & \eta_{H+}(\tau) \triangleq \int_{0}^{1} \min \left\{\frac{\tau}{T}, \frac{H(v)}{T}\right\} \mathrm{d} F(v), \text { and } \\
\Lambda_{H-}(x) \triangleq \lambda T \eta_{H-}(x), \quad \text { for } \quad & \eta_{H-}(x) \triangleq \int_{x}^{1} \frac{H(v)}{T} \mathrm{~d} F(v)=\bar{F}(x)-\eta_{H}(x),
\end{array}
$$

where $\bar{F}(x)$ stands for the tail distribution of the valuations. Note $\Lambda_{H+}(\tau)$ is the average number of buyers selecting the fixed-price channel during $[0, \tau]$, and $\eta_{H+}(\tau)$ is the corresponding fraction of arrivals in this category. Since buyers arrive according to a Poisson process of rate $\lambda$, it follows that

$$
\mathbb{P}\left(Q_{\tau}>0 \mid H\right)=\mathbb{P}\left(B\left(\Lambda_{H+}(\tau)\right) \leq Q_{0}-1\right)=\sum_{k=0}^{Q_{0}-1} \frac{\left(\Lambda_{H+}(\tau)\right)^{k} \exp \left(-\Lambda_{H+}(\tau)\right)}{k !}
$$

On the other hand, $\Lambda_{H_{-}}(x)$ represents the average number of fixed-price buyers (i.e. those below the threshold $H$ ) with valuation greater than or equal to $x$, and $\eta_{H_{-}}(x)$ is the fraction of arrivals with valuation greater than or equal to $x$ who go for the fixed-price channel.

The random variable $B\left(\Lambda_{H_{-}}(x)\right)$ represents the number of fixed-price buyers with valuation greater than or equal to $x$. Again, given that customers arrive according to a Poisson process with rate $\lambda$, we have that $B\left(\Lambda_{H_{-}}(x)\right)$ has a Poisson distribution with mean $\Lambda_{H_{-}}(x)$. One important member of this family of random variables is $B\left(\Lambda_{H_{-}}(0)\right)$, which represents the total number of buyers that have selected the fixed-price channel. Therefore, we can define the number of units left for the auction as $Q_{T}=\left(Q_{0}-B\left(\Lambda_{H_{-}}(0)\right)\right)^{+}$. From this observation and condition (7) we obtain

$$
\begin{aligned}
& \mathbb{P}\left(P_{H}(v)<x\right)=\sum_{k=1}^{Q_{0}} \mathbb{P}\left(B\left(\Lambda_{H}(x)\right)+\mathbb{1}(x \leq v) \leq k\right) \mathbb{P}\left(Q_{T}=k\right) \\
& =\sum_{k=1}^{Q_{0}} \sum_{n=0}^{k-\mathbb{1}(x \leq v)} \frac{\left(\Lambda_{H}(x)\right)^{n} \exp \left(-\Lambda_{H}(x)\right)}{n !} \frac{\left(\Lambda_{H-}(0)\right)^{Q_{0}-k} \exp \left(-\Lambda_{H-}(0)\right)}{\left(Q_{0}-k\right) !} \\
& =\left[\sum_{k=0}^{Q_{0}-1} \frac{\left(\Lambda_{H}(x)+\Lambda_{H-}(0)\right)^{k}}{k !}+\mathbb{1}(x>v) \frac{\left(\Lambda_{H}(x)+\Lambda_{H-}(0)\right)^{Q_{0}}-\left(\Lambda_{H-}(0)\right)^{Q_{0}}}{Q_{0} !}\right] \exp \left(-\left(\Lambda_{H}(x)+\Lambda_{H-}(0)\right)\right)
\end{aligned}
$$


We note that for $x \leq v$, the distribution of $P_{H}(v)$ reduces to

$$
\mathbb{P}\left(P_{H}(v)<x\right)=\sum_{k=0}^{Q_{0}-1} \frac{\left(\Lambda_{H}(x)+\Lambda_{H-}(0)\right)^{k}}{k !} \exp \left(-\left(\Lambda_{H}(x)+\Lambda_{H-}(0)\right)\right)=\mathbb{P}\left(B\left(\Lambda_{H}(x)+\Lambda_{H-}(0)\right) \leq Q_{0}-1\right) .
$$

To get some intuition about this condition (25), note that $\Lambda_{H}(x)+\Lambda_{H_{-}}(0)$ represents the average number of buyers that either enter the auction bidding more than $x$ (first summand) or buy directly the object from the fixed-price channel (second summand).

We are now ready to characterize the best-response mapping $\mathcal{R}$ in this dual channel case. Consider buyer $\tau$ arriving at time $\tau$ with valuation $v_{\tau}$. If $v_{\tau} \leq \hat{P}$ then the auction is his only profitable channel and so he enters the auction independently of $\tau$. On the other hand, if $v_{\tau}>\hat{P}$ then both channels are potentially profitable. If he decides to buy a unit through the fixed-price channel, his expected utility is zero if $Q_{\tau}=0$ (that is, there are no units left) or equals $u\left(\tau, \tau, v_{\tau}-\hat{P}\right)$ if $Q_{\tau}>0$. Thus, the expected utility if he selects the fixed-price channel is given by $\left(v_{\tau}-\hat{P}\right) \mathbb{P}\left(Q_{\tau}>0 \mid H\right)$. On the other hand, if buyer $\tau$ decides to bid and gets one object, then his utility is $u\left(\tau, T, v_{\tau}-P_{H}\left(v_{\tau}\right)\right)$, and zero otherwise. ${ }^{10}$ Therefore, buyer $\tau$ enters the auction if his expected utility from bidding exceeds his expected utility from the fixed-price channel. From the exponentially discounted utility function (1) that we consider, this participation condition is equivalent to

$$
\exp (-w(T-\tau))\left(v_{\tau}-\mathrm{E}\left[P_{H}\left(v_{\tau}\right) \mid P_{H}\left(v_{\tau}\right)<v_{\tau}\right]\right) \operatorname{Pr}\left(P_{H}\left(v_{\tau}\right)<v_{\tau}\right) \geq\left(v_{\tau}-\hat{P}\right) \mathbb{P}\left(Q_{\tau}>0 \mid H\right)
$$

which we can rewrite for the case $v_{\tau}>\hat{P}$ in the more convenient form (see equation (28)):

$$
\frac{1}{v_{\tau}-\hat{P}} \int_{0}^{v_{\tau}} \mathbb{P}\left(P_{H}\left(v_{\tau}\right)<x\right) \mathrm{d} x \geq \exp (w(T-\tau)) \mathbb{P}\left(Q_{\tau}>0 \mid H\right) .
$$

Condition (24) implies that for every $H \in \mathcal{H}$ the function $\mathbb{P}\left(Q_{\tau}>0 \mid H\right)$ is continuous and nonincreasing in $\tau \in[0, T]$. Therefore, the function $\mathcal{F}(H)(\tau) \triangleq \exp (w(T-\tau)) \mathbb{P}\left(Q_{\tau}>0 \mid H\right)$ is monotonically decreasing in $\tau$ and admits a continuous decreasing inverse function $\mathcal{F}(H)^{-1}$ in the domain $\left[\mathbb{P}\left(Q_{T}>0 \mid H\right), \exp (w T)\right]$. We find convenient to (continuously) extend this domain of $\mathcal{F}(H)^{-1}$ to the entire $\mathbb{R}^{+}$as follows:

$$
\mathcal{F}(H)^{-1}(x)=T, x \in\left[0, \mathbb{P}\left(Q_{T}>0 \mid H\right)\right] \quad \text { and } \quad \mathcal{F}(H)^{-1}(x)=0, x \geq \exp (w T) .
$$

Although a closed-form expression for $\mathcal{F}(H)^{-1}$ is not available, its existence is all what we need to establish the following result.

Proposition 4 In the dual channel case, for any strategy $H \in \mathcal{H}$, the corresponding best-response participation strategy $\mathcal{R}(H) \in \mathcal{H}$ satisfies

$$
\mathcal{R}(H)\left(v_{\tau}\right)=\left\{\begin{array}{cl}
0 & \text { if } v_{\tau} \in[0, \hat{P}] \\
\mathcal{F}(H)^{-1}\left(\frac{\int_{0}^{v_{\tau}} \mathbb{P}\left(P_{H}\left(v_{\tau}\right)<x\right) d x}{v_{\tau}-\hat{P}}\right) & \text { if } v_{\tau} \in(\hat{P}, 1] .
\end{array}\right.
$$

\footnotetext{
${ }^{10}$ Note that in this dual channel setting, the seller clears $\min \left\{Q_{0}, B(\lambda T)\right\}$ units through both channels by time $T$.
} 
The proof of the Proposition is omitted as it follows directly from the participation condition (26) and the extended definition of $\mathcal{F}(H)^{-1}$ above. Only the continuity of $\mathcal{R}(H)\left(v_{\tau}\right)$ at $v_{\tau}=\hat{P}$, as it is required by the condition $\mathcal{R}(H) \in \mathcal{H}$, deserves some attention. For this, note that for all $H \in \mathcal{H}$ we have that

$$
\lim _{v_{\tau} \downarrow \hat{P}} \frac{\int_{0}^{v_{\tau}} \mathbb{P}\left(P_{H}\left(v_{\tau}\right)<x\right) \mathrm{d} x}{v_{\tau}-\hat{P}} \rightarrow+\infty .
$$

Continuity at $\hat{P}$ now follows from the fact that $\mathcal{F}(H)^{-1}(x)=0$ for all $x \geq \exp (w T)$. Using a similar argument, we also note that for every $H \in \mathcal{H}$ there is a $v_{H}>\hat{P}$ such that $\mathcal{R}(H)(v)=0$ for all $v \in\left[\hat{P}, v_{H}\right]$. As in equation (14), we define

$$
\tilde{v} \triangleq \inf _{H \in \mathcal{H}}\left\{v_{H}\right\}
$$

We can get a lower bound on $\tilde{v}$ from the fact that

$$
\frac{\int_{0}^{v_{\tau}} \mathbb{P}\left(P_{H}\left(v_{\tau}\right)<x\right) \mathrm{d} x}{v_{\tau}-\hat{P}} \geq \frac{\int_{0}^{v_{\tau}} \mathbb{P}\left(B(\lambda T(1-F(\hat{P}))) \leq Q_{0}-1\right) \mathrm{d} x}{v_{\tau}-\hat{P}}=\frac{v_{\tau} \mathbb{P}\left(B(\lambda T(1-F(\hat{P}))) \leq Q_{0}-1\right)}{v_{\tau}-\hat{P}} .
$$

The lower bound $\underline{v}$ is obtained by solving

$$
\frac{v_{\tau} \mathbb{P}\left(B(\lambda T(1-F(\hat{P}))) \leq Q_{0}-1\right)}{v_{\tau}-\hat{P}}=\exp (w T)
$$

that is,

$$
\underline{v}=\frac{\exp (w T) \hat{P}}{\exp (w T)-\mathbb{P}\left(B(\lambda T(1-F(\hat{P}))) \leq Q_{0}-1\right)} .
$$

As in the single channel case, the existence of $\tilde{v}>\hat{P}$ guarantees that the best-response strategy $\mathcal{R}(H)$ is $K$-Lipschitz continuous for an appropriate constant $K$. Therefore, we can redefine the space of strategies to be

$$
\mathcal{H} \triangleq\{H:[0,1] \rightarrow[0, T] \text { s.t. } H \text { is } K \text {-Lipschitz continuous and } H(v)=0 \text { in } v \in[0, \tilde{v}]\}
$$

The following result formalizes this claim and proves the existence of a symmetric participation equilibrium (SPE) for this dual channel case.

Theorem 4 For the exponential utility function (1) and for all $H \in \mathcal{H}$, there is a positive constant $K$ (independent of $H$ ) such that the best-response strategy $\mathcal{R}(H)(v)$ is a K-Lipschitz continuous function that satisfies $\mathcal{R}(H)(v)=0$ for all $v \in[0, \tilde{v}]$. In addition, the best-response mapping $\mathcal{R}$ is continuous in $\mathcal{H}$ equipped with the uniform norm, and so a symmetric equilibrium always exists in $\mathcal{H}$.

Again, like in the end of Section 3.2, we point out the existence of $v_{H^{*}}$ and its implication: there is always a range of buyer valuations above the list price $\hat{P}$, such that those buyers will join the auction regardless their arrival time. 


\subsection{Asymptotic analysis}

Following Section 3.3, here we analyze the limiting regime for the dual channel setting when both the initial number of units $Q_{0}$ and the arrival rate $\lambda$ grow proportionally large (see equation (15)). The auction price for instance $n$ given that a bidder with valuation $v$ enters the auction is $P_{H}^{n}(v)$, and the final random number of units to auction is $Q_{T}^{n}$. Next proposition characterizes the asymptotic regime:

Theorem 5 Suppose the participation strategy $H(v)$ and the static price $\hat{P}$ are given. Then, in the limit as $n \rightarrow \infty$ :

i) The re-scaled number of units $Q_{T}^{n} / n$ to sell through the auction converges weakly to a constant $Q_{T} \triangleq\left(Q_{0}-\lambda T \eta_{H-}(0)\right)^{+}$.

ii) If a final auction takes place (i.e. $\left.Q_{T}>0\right)$, its price $P_{H}^{n}(v)$ converges weakly to a constant $P_{H}^{\infty} \triangleq \min \left\{v \in[0,1]: \eta_{H}(v) \leq \rho-\eta_{H-}(0)\right\}$, where $\rho$ is defined in equation (16).

Following the argument in Section 3.3, in order to determine the value of the auction price $P_{H}$ and the corresponding participation strategy $H(v)$, we have to impose the equilibrium condition $\mathcal{R}\left(H^{*}\right)=H^{*}$. Since $\mathcal{R}(H)(v)=0$ for all $v<\hat{P}$, we must have $H^{*}(v)=0$ in $v \in[0, \hat{P})$. In words, buyers with valuation smaller than the posted price $\hat{P}$ have no other choice but entering the auction. To describe the behavior of $H^{*}(v)$ in $v \in[\hat{P}, 1]$ we need to distinguish two cases:

Case 1: Suppose that the initial supply of units is limited in the sense that $\rho \leq 1-F(\hat{P})$. In this situation, buyers with valuation greater than $\hat{P}$ have no incentive to enter the auction since the auction price is guaranteed to be greater than or equal to $\hat{P}$. Therefore, the resulting participation strategy is $H^{*}(v)=T$ for all $v \geq \hat{P}$ and the auction never takes place since all the units will be bought at the posted price (i.e. $Q_{T}=0$ ).

We note that $H^{*}(v)=T \mathbb{1}(v \geq \hat{P})$ is not the only SPE in this case. In fact, let us define $\tau^{*} \triangleq T \rho(1-F(\hat{P}))^{-1}$. Then, any $H$ of the form $H(v)=\left(\tau^{*}+h(v)\right) \mathbb{1}(v \geq \hat{P})$ for an arbitrary nonnegative and bounded function $h(v) \leq T-\tau^{*}$ is a SPE. In fact, for such an $H$ the initial $Q_{0}$ units will be depleted by time $\tau^{*}$ (i.e. $Q_{\tau^{*}}=0$, since $\tau^{*} \lambda(1-F(\hat{P}))=Q_{0}$ ). Therefore, any buyer arriving after $\tau^{*}$ will never get a unit and so he becomes indifferent between the two channels.

Case 2: Suppose that initial supply is abundant in the sense that $\rho>1-F(\hat{P})$. In this case, $Q_{T}>0$ and some buyers with valuation smaller than $\hat{P}$ get units through the auction. It is not hard to see that in this case the auction price is given by $P_{H^{*}}=F^{-1}(1-\rho)$. Therefore, buyer $\tau$ arriving at time $\tau$ with valuation $v_{\tau} \geq \hat{P}$ enters the auction only if $v_{\tau}-\hat{P} \leq \exp (-w(T-\tau))\left(v_{\tau}-P_{H^{*}}\right)$. We conclude that in this abundant case the unique $\operatorname{SPE} H^{*}(v)$ is given by:

$$
H^{*}(v)=\left\{\begin{array}{cl}
0 & \text { if } v \in\left[0, v_{H^{*}}\right] \\
T-\frac{1}{w} \ln \left(\frac{v-P_{H^{*}}}{v-\hat{P}}\right) & \text { if } v \in\left[v_{H^{*}}, 1\right]
\end{array} \quad \text { where } v_{H^{*}}=\min \left\{\frac{\hat{P} \exp (w T)-P_{H^{*}}}{\exp (w T)-1}, 1\right\}\right.
$$


Figure 6 compares the optimal asymptotic strategy $H^{*}(\infty, \rho)$ to four optimal participation strategies $H^{*}\left(Q_{0}, \rho\right)\left(Q_{0}=1,5,10,20\right)$ for the case of $\rho=0.5$. As in the single channel case, the asymptotic strategy is almost identical to the optimal strategy for values of $Q_{0}$ greater than 10 units. The table on the right compares the expected value of the auction price $P_{H}$ and the approximation $P_{H}^{\text {Approx }}$ obtained using the asymptotic participation strategy $H^{*}(\infty, 0.5)$. Similarly to the single auction channel, the asymptotic approximation is very accurate even for small values of $Q_{0}$.

\section{Asymptotic Approximation: Computational Experiments}

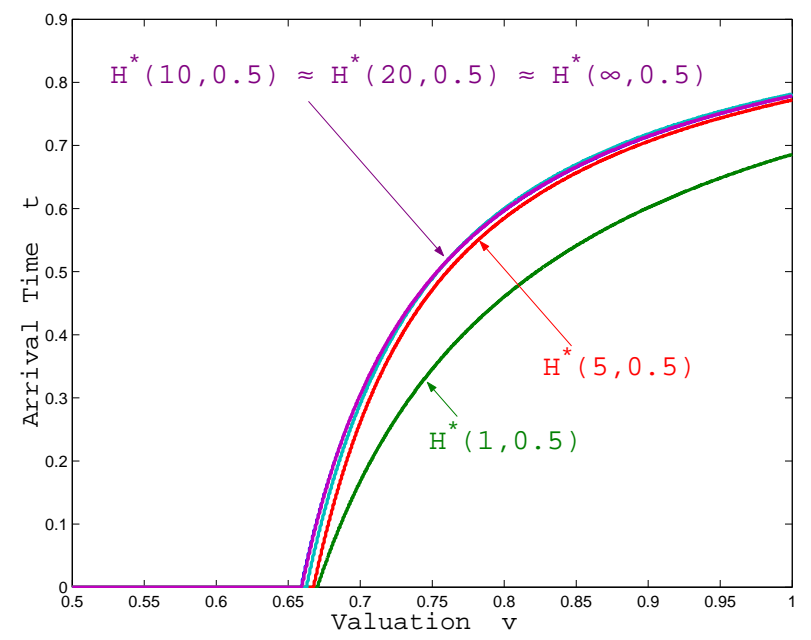

\begin{tabular}{||c|c|c|c||}
\hline \hline$Q_{0}$ & $P_{H}$ & $P_{H}^{\text {Approx }}$ & Error \\
\hline 1 & 0.2793 & 0.2834 & $1.428 \%$ \\
\hline 5 & 0.4175 & 0.4184 & $0.213 \%$ \\
\hline 10 & 0.4539 & 0.4540 & $0.034 \%$ \\
\hline 20 & 0.4760 & 0.4760 & $0.002 \%$ \\
\hline \hline
\end{tabular}

Figure 6: Asymptotic approximation for the case with valuations uniformly distributed in $[0,1]$, and $T=w=1$, $\hat{P}=0.6$ and $\rho=0.5$. In this case, the asymptotic price is $P_{H^{*}}^{\infty}=1-\rho=0.5$.

As in the single auction channel, we conclude this section specializing the asymptotic results to the case of uniformly distributed valuations.

\section{Example: Uniform Distribution Case.}

Suppose buyers' valuations are uniformly distributed in $[0,1]$. Under this assumption, we will characterize the auction price $P_{H^{*}}$ as well as the number of units sold in the auction $Q_{H^{*}}$, the cumulative number of units sold in the fixed-price channel during $[0, T] Q_{F^{*}}$, and the corresponding rate $\lambda_{F^{*}}(t)$ at which these units are sold. In the asymptotic regime under consideration we have that

$$
Q_{F^{*}}=\int_{0}^{T} \lambda_{F^{*}}(t) \mathrm{d} t
$$

Depending on the values of $\rho$ and $\hat{P}$, we distinguish two cases.

Limited-Supply Case: $\rho \leq 1-\hat{P}$. In this case, all units are depleted through the fixed-price channel, that is, $Q_{F^{*}}=Q_{0}$ and $Q_{H^{*}}=0$. In addition, the fixed-price channel demand rate satisfies

$$
\lambda_{F^{*}}(t)=\lambda(1-\hat{P}) \mathbb{1}\left(t \leq \tau^{*}\right), \quad t \in[0, T]
$$


where $\tau^{*}=\frac{Q_{0}}{\lambda(1-\hat{P})} \leq T$ is the time at which all $Q_{0}$ units are sold.

Abundant-Supply Case: $\rho>1-\hat{P}$. In this case, there is a positive number of units that are sold through the auction at a price $P_{H^{*}}=(1-\rho)^{+}$. The number of units auctioned and sold through the fixed-price channel are

$$
Q_{H^{*}}=\lambda T \eta_{H^{*}}\left(P_{H^{*}}\right) \quad \text { and } \quad Q_{F^{*}}=\lambda T\left(1-\eta_{H^{*}}\left(P_{H^{*}}\right)\right)
$$

respectively, where

$$
\eta_{H^{*}}\left(P_{H^{*}}\right)=\int_{P_{H^{*}}}^{1}\left(1-\left[1-\frac{1}{w T} \ln \left(\frac{v-(1-\rho)^{+}}{v-\hat{P}}\right)\right]^{+}\right) \mathrm{d} v .
$$

In this case, the fixed-price channel demand rate satisfies

$$
\begin{aligned}
\lambda_{F^{*}}(t) & =\lambda H^{*-1}(t) \\
& =\lambda\left[1-\frac{\hat{P} \exp (w(T-t))-(1-\rho)^{+}}{\exp (w(T-t))-1}\right]^{+} .
\end{aligned}
$$

\subsection{Seller's optimization problem}

For this dual channel setting, the seller must solve (numerically) a more complex problem than the one in Section 3.4, since she also controls the list price $\hat{P}$. Again, for simplicity and provided the accuracy of the approximation in Section 4.2, we will work on the asymptotic regime, assuming that the original distribution of valuations is $F \triangleq \operatorname{Unif}[0,1]$. We also reverse the scaling in (3), that is,

$$
\lambda \rightarrow \lambda\left(1-v^{\mathrm{R}}\right), \quad \rho \rightarrow \frac{Q_{0}}{\lambda T\left(1-v^{\mathrm{R}}\right)}=\frac{\rho}{1-v^{\mathrm{R}}}, \quad \hat{P} \rightarrow \frac{\hat{P}-v^{\mathrm{R}}}{1-v^{\mathrm{R}}},
$$

to formulate the seller's problem in terms of the original data. This problem consists on finding a reservation price $v^{\mathrm{R}}$, a posted price $\hat{P}$, and possibly an auction duration $T$, that maximize the seller's revenue, which we assume is exponentially discounted over time at rate $\alpha$. Note that the seller can restrict the optimization to those cases in which $v^{\mathrm{R}} \leq \hat{P}$, otherwise the auction is trivially dominated by the fixed-price channel. Based on the results in Section 4.2, we distinguish two cases.

Limited-Supply Case: If the seller chooses a posted-price $\hat{P}$ such that $\rho \leq 1-\hat{P}$ then independently of the reservation price $v^{\mathrm{R}}$ the auction never takes place; all units are sold through the fixed-price channel. The seller's revenue function in this case is given by

$V_{D}(\hat{P}, T)=\int_{0}^{\tau^{*}} \hat{P} \exp (-\alpha t) \lambda(1-F(\hat{P})) \mathrm{d} t=\frac{\hat{P} \lambda(1-F(\hat{P}))}{\alpha}\left(1-\exp \left(-\alpha \tau^{*}\right)\right)$, where $\tau^{*}=\frac{Q_{0}}{\lambda(1-\hat{P})}$,

which leads to the following constrained optimization: $V_{D}^{*} \triangleq \max _{\hat{P}, T}\left\{V_{D}(\hat{P}, T): 0 \leq \hat{P} \leq 1-\frac{Q_{0}}{\lambda T}\right\}$. 
Abundant-Supply Case: In this case, the seller chooses a posted price $\hat{P}$ such that $\rho>1-\hat{P}$. In this case a final auction takes place with outcome price $P_{H^{*}}=\max \left\{v^{\mathrm{R}},(1-\rho)^{+}\right\}$. The revenue just from the auction is:

$$
V_{D_{A}}\left(v^{\mathrm{R}}\right)=\lambda T \eta_{H^{*}}\left(P_{H^{*}}\right) P_{H^{*}} \exp (-\alpha T)
$$

The calculation of the revenue from the list price channel requires inverting the scaling for the fixedprice channel demand rate in $(27)$, that is,

$$
\lambda_{F^{*}}(t)=\lambda\left[1-v^{\mathrm{R}}-\frac{\left(\hat{P}-v^{\mathrm{R}}\right) \exp (w(T-t))-\left(1-\rho-v^{\mathrm{R}}\right)^{+}}{\exp (w(T-t))-1}\right]^{+} .
$$

The revenue obtained from the list price channel is therefore:

$$
V_{D_{L P}}(\hat{P})=\hat{P} \int_{0}^{1} \exp (-\alpha t) \lambda_{F^{*}}(t) \mathrm{d} t
$$

where the integral represents the aggregated cumulative discount for the list price buyers (i.e. those customers below the curve $\left.H^{*}\right)$.

Figure 7 shows the expected seller's revenue as a function of the list price $\hat{P}$, given that the reservation price of the auction has been optimized for every $\hat{P}$. The parameters considered are: $T=w=1, \alpha=2$, and $\lambda=10$. In Case a), the list price to post must be marginally above 0.6. For prices below, there is no auction (case 1 in Section 4.2), and $\hat{P}$ determines the revenues. For prices above, there are some remaining units at $T$, and the auction takes place (case 2 in Section 4.2). Moreover, for $\hat{P}>0.84$, no customers go to the posted price channel. In Case b), the optimal list price to post is $\hat{P}=0.5$ : For list prices in the range [0, 0.92], there is no auction; and for list prices above 0.92 , nobody goes for the list price.

\section{Concluding remarks}

In this paper, we have proposed a model to analyze the problem faced by a seller when designing a single channel online auction, or when managing a dual online auction and list price channel. The key to build this model is understanding the strategic behavior of the bidders, provided they choose to either join the auction or buy the product at the posted price.

The private information of the buyers has two dimensions: the arrival time, and the private value for one of the units being offered. For a discounted utility function, we showed that their participation equilibrium strategy is of the threshold type, that is, a buyer will join the auction if and only if his arrival time is higher than a function of his own valuation. Of course, for buyers with values below the list price, the optimal strategy is always to participate in the auction. For buyers with higher values, the threshold is nondecreasing in the own valuation. Interestingly, we found that there is always a range of values above the fixed price for which it is also optimal to go for the auction, regardless the arrival time. 


\section{Optimization of the List Price for the Dual Channel Case}

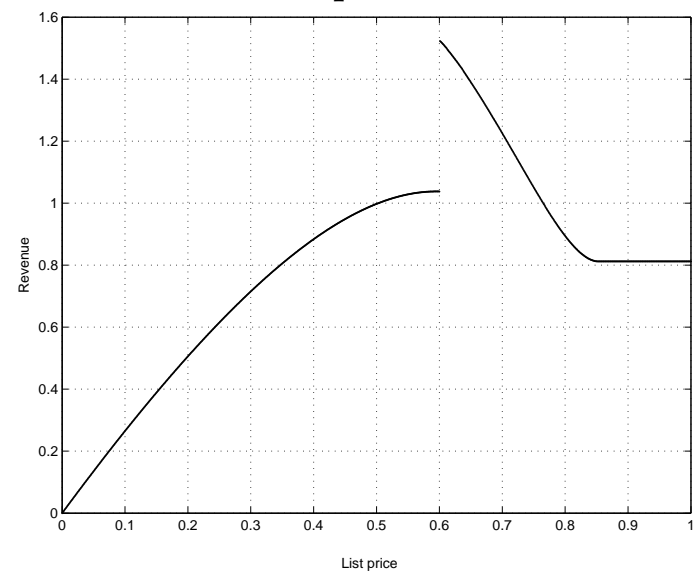

Case (a): $Q_{0}=4$

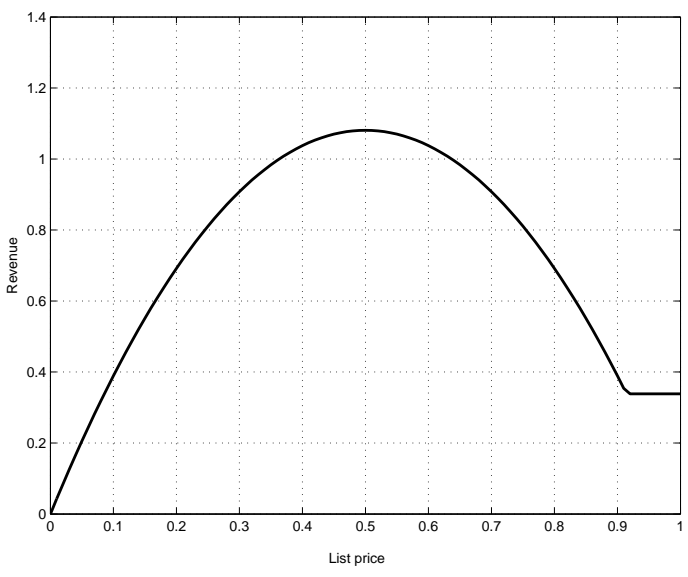

Case (b): $Q_{0}=10$

Figure 7: Seller's revenue as a function of the list price $\hat{P}$, auction duration $T=1$, original valuations with distribution $F \triangleq \operatorname{Unif}[0,1], \lambda=10, w=1$, and $\alpha=2$. In case (a), the seller has to announce a list price marginally above 0.6. In case (b), the optimal list price is $\hat{P}=0.5$.

We proposed a contraction algorithm in a function space to find this threshold, and proved that it is convergent under some conditions. When these conditions do not hold, we managed to find the fixed point of the algorithm by slightly perturbing the values of each iteration.

To overcome the computationally intensive feature of this procedure, we provided asymptotic analysis for both settings. In the limiting regimes, we got very simple limiting threshold functions which turned out to be indeed good approximations of the true equilibrium functions, according to our numerical experiments. Given this accuracy, we plugged in this limiting threshold in the seller's optimization problem, and numerically solved for few instances. Because of the number of parameters involved in the formulations (number of units, auction duration, auction reservation price, and list price in the dual channel case), we have just provided few examples as simple illustrations of the potential use of this analysis.

We believe that the techniques used to derive the equilibrium participation strategy here, in particular the asymptotic analysis, can also be used when extending our model to incorporate the number of remaining units as part of the information structure of the bidders. That would mean adding one coordinate to the threshold, leading to a three-dimensional surface, but we have not explored this direction in detail.

Other possible extensions are related to the seller's optimization problem. In our formulation, we have included the capital cost. But for example, in the dual channel case, one can easily add some holding cost for keeping the units until the end of the time horizon, such that the seller has an incentive to give more units through the list price channel. One could also add a penalty cost for keeping units at the end of the horizon, or a shortage cost for not being able to serve a customer if the units are 
depleted before the time scheduled for the auction.

Acknowledgements. The authors would like to thank Victor Araman (New York University), Dorothée Honhon (New York University), and Costis Maglaras (Columbia University) for helpful discussions. 


\section{Appendix}

The following Lemma will be used a few times in this appendix.

Lemma 2 Let $B(x)$ a Poisson random variable mean $x>0$. Then, for a nonnegative integer $n$

$$
\frac{d}{d x} \mathbb{P}(B(x) \leq n)=-\mathbb{P}(B(x)=n) .
$$

Proof:

$$
\begin{aligned}
\frac{\mathrm{d}}{\mathrm{d} x} \mathbb{P}(B(x) \leq n) & =\sum_{k=0}^{n} \frac{\mathrm{d}}{\mathrm{d} x}\left(\frac{(x)^{k} \exp (-x)}{k !}\right) \\
& =\sum_{k=1}^{n} \frac{k(x))^{k-1} \exp (-x)}{k !}-\sum_{k=0}^{n} \frac{(x)^{k} \exp (-x)}{k !} \\
& =-\frac{(x)^{n} \exp (-x)}{n !}=-\mathbb{P}(B(x)=n)
\end{aligned}
$$

\section{Proof of Proposition 1}

Using the fact that $P_{H}\left(v_{\tau}\right)$ is a random variable with positive mass at $v^{\mathrm{R}}=0$ and the properties of conditional expectation, we have that

$$
\begin{aligned}
& \mathrm{E}\left[P_{H}\left(v_{\tau}\right) \mid P_{H}\left(v_{\tau}\right)<\hat{P}\right] \\
= & v^{\mathrm{R}} \mathbb{P}\left(P_{H}\left(v_{\tau}\right)=v^{\mathrm{R}} \mid P_{H}\left(v_{\tau}\right)<\hat{P}\right)+\mathrm{E}\left[P_{H}\left(v_{\tau}\right) \mid v^{\mathrm{R}}<P_{H}\left(v_{\tau}\right)<\hat{P}\right] \mathbb{P}\left(P_{H}\left(v_{\tau}\right)>v^{\mathrm{R}} \mid P_{H}\left(v_{\tau}\right)<\hat{P}\right) \\
= & \left(\int_{0}^{\hat{P}} \mathbb{P}\left(P_{H}\left(v_{\tau}\right) \geq x \mid 0<P_{H}\left(v_{\tau}\right)<\hat{P}\right) \mathrm{d} x\right) \mathbb{P}\left(P_{H}\left(v_{\tau}\right)>0 \mid P_{H}\left(v_{\tau}\right)<\hat{P}\right) \\
= & \int_{0}^{\hat{P}} \frac{\mathbb{P}\left(x \leq P_{H}\left(v_{\tau}\right)<\hat{P}\right)}{\mathbb{P}\left(P_{H}\left(v_{\tau}\right)<\hat{P}\right)} \mathrm{d} x
\end{aligned}
$$

Combining this expression and (9), after some algebra we get the participation constraint:

$$
\begin{aligned}
\tau & \geq T-\frac{1}{w} \ln \left(\left[\hat{P} \mathbb{P}\left(P_{H}\left(v_{\tau}\right)<\hat{P}\right)-\int_{0}^{\hat{P}} \mathbb{P}\left(x \leq P_{H}\left(v_{\tau}\right)<\hat{P}\right) \mathrm{d} x\right] \frac{1}{v_{\tau}-\hat{P}}+1\right) \\
& =T-\frac{1}{w} \ln \left(1+\int_{0}^{\hat{P}} \frac{\mathbb{P}\left(P_{H}\left(v_{\tau}\right)<x\right)}{v_{\tau}-\hat{P}} \mathrm{~d} x\right) \triangleq h_{H}\left(v_{\tau}\right),
\end{aligned}
$$

which proves (11). From this last inequality, it follows that the right-hand side $h_{H}\left(v_{\tau}\right)$ is the natural candidate for best-response strategy for buyer $\tau$ with valuation $v_{\tau}>\hat{P}$. However, for an arbitrary $H \in \mathcal{H}, h_{H}\left(v_{\tau}\right)$ is not guaranteed to be nonnegative and so $h_{H}$ is not necessarily a well-defined participation strategy in $\mathcal{H}$. Fortunately, note that we can correct this problem by simply setting the best-response strategy $\mathcal{R}(H)\left(v_{\tau}\right)=\left(h_{H}\left(v_{\tau}\right)\right)^{+}$which is equivalent to (12) and consistent with (9). 


\section{Proof of Proposition 2}

Note that we only need to prove the $K$-Lipschitz property of $\mathcal{R}(H)(v)$; the rest of the proposition follows directly from the definition of $\tilde{v}$.

To prove that $\mathcal{R}(H)(v)$ is $K$-Lipschitz continuous, we will make use of the following lemma (not hard to prove).

Lemma 3 For arbitrary reals $a, b$, and $c: \quad\left|[a-b]^{+}-[a-c]^{+}\right| \leq|b-c|$. For arbitrary nonnegative reals $x, y \geq 1: \quad|\ln (x)-\ln (y)| \leq|x-y|$.

Recall from the definition of $v_{H}$ that $\mathcal{R}(H)(v)=0$ for all $v \in\left[0, v_{H}\right]$. So, we can concentrate on proving the $K$-Lipschitz property on the interval $\left[v_{H}, 1\right]$. In this range, condition (12) implies that

$$
\mathcal{R}(H)(v)=\left[T-\frac{1}{w} \ln \left(Z_{H}(v)\right)\right]^{+} \text {where } Z_{H}(v) \triangleq 1+\int_{0}^{\hat{P}} \frac{\mathbb{P}\left(P_{H}(v)<x\right)}{v-\hat{P}} \mathrm{~d} x .
$$

So based on the previous lemma we have that for arbitrary $v_{1}, v_{2} \in\left[v_{H}, 1\right]$

$$
\begin{aligned}
\left|\mathcal{R}(H)\left(v_{1}\right)-\mathcal{R}(H)\left(v_{2}\right)\right| & =\left|\left[T-\frac{1}{w} \ln \left(Z_{H}\left(v_{1}\right)\right)\right]^{+}-\left[T-\frac{1}{w} \ln \left(Z_{H}\left(v_{2}\right)\right)\right]^{+}\right| \\
& \leq \frac{1}{w}\left|\ln \left(Z_{H}\left(v_{1}\right)\right)-\ln \left(Z_{H}\left(v_{2}\right)\right)\right| \\
& \leq \frac{1}{w}\left|Z_{H}\left(v_{1}\right)-Z_{H}\left(v_{2}\right)\right|
\end{aligned}
$$

From the definition of $Z_{H}(v)$ and the fact that $\int_{0}^{\hat{P}} \mathbb{P}\left(P_{H}(v)<x\right) \mathrm{d} x$ is independent of $v$ for $v \geq v_{H}>\hat{P}$ (since bidders with valuation in this range bid $b(v)=\hat{P}$ independent of $v$ ), we have that

$$
\begin{aligned}
\left|Z_{H}\left(v_{1}\right)-Z_{H}\left(v_{2}\right)\right| & =\left|\int_{v_{1}}^{v_{2}} \frac{\mathrm{d}}{\mathrm{d} v} Z_{H}(v) \mathrm{d} v\right| \\
& \leq\left|\int_{v_{1}}^{v_{2}}\left[-\int_{0}^{\hat{P}} \frac{\mathbb{P}\left(P_{H}(v)<x\right)}{(v-\hat{P})^{2}} \mathrm{~d} x\right] \mathrm{d} v\right| \\
& \leq\left|\int_{v_{1}}^{v_{2}}\left[\frac{\hat{P}}{(\tilde{v}-\hat{P})^{2}}\right] \mathrm{d} v\right| \quad(\text { using } \tilde{v} \leq v \leq 1) \\
& =\left[\frac{\hat{P}}{(\tilde{v}-\hat{P})^{2}}\right]\left|v_{1}-v_{2}\right| .
\end{aligned}
$$

We conclude that for arbitrary $v_{1}, v_{2} \in\left[v_{H}, 1\right]$

$$
\left|\mathcal{R}(H)\left(v_{1}\right)-\mathcal{R}(H)\left(v_{2}\right)\right| \leq \frac{1}{w}\left[\frac{\hat{P}}{(\tilde{v}-\hat{P})^{2}}\right]\left|v_{1}-v_{2}\right| \triangleq K\left|v_{1}-v_{2}\right| .
$$

The constant $K$ is guaranteed to by finite since $\tilde{v} \geq \underline{v}>\hat{P}$.

\section{Proof of Theorem 1}


To prove that $\mathcal{H}$ has the fixed-point property, we apply the Schauder-Tychonoff Fixed-Point Theorem (see Cheney [9, Chapter 7] for details). For this, we need to show that $\mathcal{H}$ is a compact convex set. Convexity is immediate from the definition of $\mathcal{H}$. To check compactness, we apply the ArzelàAscoli Theorem II (Cheney [9, Chapter 7]), that is, we need to show that $\mathcal{H}$ is closed, bounded, and equicontinuous. Take a sequence $\left\{H^{n}\right\}_{n \geq 1}$ of strategies in $\mathcal{H}$ that converges pointwise to $H$. For all $v \in[0, \tilde{v}], H^{n}(v)=0$ and so $H(v)=0$ as well. In addition, to verify the $K$-Lipschitz property of $H$ note that from Proposition 2, for $n \geq 1$ and $v_{1}, v_{2} \in[0,1]$

$$
\left|H^{n}\left(v_{1}\right)-H^{n}\left(v_{2}\right)\right| \leq K\left|v_{1}-v_{2}\right|
$$

By the continuity of the absolute value and the point-wise convergence of $H^{n}$ to $H$ we conclude

$$
\left|H\left(v_{1}\right)-H\left(v_{2}\right)\right| \leq K\left|v_{1}-v_{2}\right|,
$$

which proves the closedness of $\mathcal{H}$. The boundedness of $\mathcal{H}$ follows from the fact $H(v) \in[0, T]$ for all $H \in \mathcal{H}$. Equicontinuity, on the other hand, follows directly from the fact that the elements of $\mathcal{H}$ are $K$-Lipschitz continuous. In fact, to prove equicontinuity we need to show that for $\epsilon>0$ there is $\delta>0$ such that:

For all $H \in \mathcal{H}$ and $v_{1}, v_{2} \in \mathcal{V}$ such that $\left|v_{1}-v_{2}\right|<\delta$ then $\left|H\left(v_{1}\right)-H\left(v_{2}\right)\right|<\epsilon$.

For this, take $\delta=\frac{\epsilon}{K}$ and use the $K$-Lipschitz continuity of $\mathcal{H}$ as follows:

For all $H \in \mathcal{H}$ and $v_{1}, v_{2} \in \mathcal{V}$ such that $\left|v_{1}-v_{2}\right|<\delta, \quad\left|H\left(v_{1}\right)-H\left(v_{2}\right)\right| \leq K\left|v_{1}-v_{2}\right|<K \delta=\epsilon$.

This proves that $\mathcal{H}$ has the fixed-point property.

We now prove that the best-response $\mathcal{R}$ mapping is continuous. Note that from the definitions of the mapping $\mathcal{R}$ and $\tilde{v}$ we just need to prove the result for the restriction of $\mathcal{R}$ to the interval $(\tilde{v}, 1]$. For the proof, we will requires the following to lemma.

Lemma 4 Let $a$ and $b$ be two nonnegative reals and $N \geq 1$ and integer. Then, there is $0 \leq \beta(N) \leq 1$ such that

$$
|\mathbb{P}(B(b) \leq N)-\mathbb{P}(B(a) \leq N)| \leq \beta(N)|b-a|
$$

Proof: Since $\mathbb{P}(B(a) \leq N)$ is a continuous and differentiable function of $a$ we have that

$$
\mathbb{P}(B(b) \leq N)=\mathbb{P}(B(a) \leq N)+\int_{a}^{b} \frac{\mathrm{d}}{\mathrm{d} x} \mathbb{P}(B(x) \leq N) \mathrm{d} x
$$

which is straightforward to prove (using Lemma 2) that is equivalent to

$$
\mathbb{P}(B(b) \leq N)=\mathbb{P}(B(a) \leq N)-\int_{a}^{b} \mathbb{P}(B(x)=N) \mathrm{d} x
$$


Therefore,

$$
\begin{aligned}
|\mathbb{P}(B(b) \leq N)-\mathbb{P}(B(a) \leq N)| & =\int_{a \wedge b}^{a \vee b} \mathbb{P}(B(x)=N) \mathrm{d} x \\
& \leq|b-a| \mathbb{P}(B(N)=N)
\end{aligned}
$$

where the inequality follows from the fact that $\mathbb{P}(B(x)=N)$ is maximized at $x=N$. So, by setting

$$
\beta(N) \triangleq \mathbb{P}(B(N)=N)
$$

the proof of the lemma is completed.

Based on Lemma 3, we have that for any $v \in(\tilde{v}, 1]$,

$$
\begin{aligned}
\left|\mathcal{R}(H)_{v}-\mathcal{R}(\tilde{H})_{v}\right|= & \mid\left[T-\frac{1}{w} \ln \left(1+\int_{0}^{\hat{P}} \frac{\mathbb{P}\left(P_{H}(v)<x\right)}{v-\hat{P}} \mathrm{~d} x\right)\right]^{+} \\
& -\left[T-\frac{1}{w} \ln \left(1+\int_{0}^{\hat{P}} \frac{\mathbb{P}\left(P_{\tilde{H}}(v)<x\right)}{v-\hat{P}} \mathrm{~d} x\right)\right]^{+} \mid \\
\leq & \frac{1}{w}\left(\int_{0}^{\hat{P}} \frac{\left|\mathbb{P}\left(P_{H}(v)<x\right)-\mathbb{P}\left(P_{\tilde{H}}(v)<x\right)\right|}{v-\hat{P}} \mathrm{~d} x\right) .
\end{aligned}
$$

Now, by Lemma 4

$$
\begin{aligned}
\left|\mathbb{P}\left(P_{H}(v)<x\right)-\mathbb{P}\left(P_{\tilde{H}}(v)<x\right)\right| & =\left|\mathbb{P}\left(B\left(\Lambda_{H}(x)\right) \leq Q_{0}-1\right)-\mathbb{P}\left(B\left(\Lambda_{\tilde{H}}(x)\right) \leq Q_{0}-1\right)\right| \\
& \leq \beta\left(Q_{0}-1\right)\left|\Lambda_{H}(x)-\Lambda_{\tilde{H}}(x)\right|=\beta\left(Q_{0}-1\right)\left|\lambda \int_{x}^{1}(\tilde{H}(y)-H(y)) \mathrm{d} F(y)\right| \\
& \leq \lambda \beta\left(Q_{0}-1\right)(1-F(x))\|H-\tilde{H}\| .
\end{aligned}
$$

Finally, from this inequality we get that for all $v \in(\tilde{v}, 1]$

$$
\begin{aligned}
\left|\mathcal{R}(H)_{v}-\mathcal{R}(\tilde{H})_{v}\right| & \leq \frac{\lambda \beta\left(Q_{0}-1\right)}{w}\left(\int_{0}^{\hat{P}} \frac{(1-F(x))}{v-\hat{P}} \mathrm{~d} x\right)\|H-\tilde{H}\| \\
& \leq \frac{\lambda \beta\left(Q_{0}-1\right)}{w}\left(\int_{0}^{\hat{P}} \frac{(1-F(x))}{\tilde{v}-\hat{P}} \mathrm{~d} x\right)\|H-\tilde{H}\|
\end{aligned}
$$

where the second inequality follow from the fact that $v \in(\tilde{v}, 1]$.

From this result, we conclude that $\mathcal{R}$ is continuous which together with the fixed-point property of the set $\mathcal{H}$ guarantee the existence of a SPE.

\section{Proof of Proposition 3}

Given the equilibrium $H^{*}$, we define $Z_{H^{*}}(v)$ by

$$
Z_{H^{*}}(v) \triangleq 1+\int_{0}^{\hat{P}} \frac{\mathbb{P}\left(P_{H^{*}}(v)<x\right)}{v-\hat{P}} \mathrm{~d} x, \quad v \in\left[v_{H^{*}}, 1\right] .
$$


Note that in the range $\left[0, v_{H^{*}}\right]$ the function $H^{*}$ is constant at 0 .

Based on the definition of the best-response mapping $\mathcal{R}$, we can write the fixed-point condition $\mathcal{R}\left(H^{*}\right)=H^{*}$ satisfied by $H^{*}(v)$ as

$$
H^{*}(v)=T-\frac{1}{w} \ln \left(Z_{H^{*}}(v)\right), \quad \text { for all } v \in\left[v_{H^{*}}, 1\right] .
$$

Taking derivative with respect to $v$ and using the fact that

$$
\chi \triangleq \int_{0}^{\hat{P}} \mathbb{P}\left(P_{H^{*}}(v)<x\right) \mathrm{d} x
$$

is independent of $v$ for $v \geq v_{H^{*}}>\hat{P}$, we get that

$$
\frac{\mathrm{d}}{\mathrm{d} v} H^{*}(v)=\frac{-1}{w} \frac{1}{Z_{H^{*}}(v)} \frac{\mathrm{d}}{\mathrm{d} v} Z_{H^{*}}(v)=\frac{\chi}{w}\left(\frac{1}{(v-\hat{P})(v-\hat{P}+\chi)}\right)>0,
$$

and we conclude that $H^{*}(v)$ is increasing in $v$ for all $v \geq v_{H^{*}}$. Finally, taking a second derivative we get

$$
\frac{\mathrm{d}^{2}}{\mathrm{~d} v^{2}} H^{*}(v)=-\frac{\chi}{w}\left(\frac{2(v-\hat{P})+\chi}{(v-\hat{P})^{2}(v-\hat{P}+\chi)^{2}}\right)<0,
$$

and we conclude that $H^{*}(v)$ is concave in the range $v \in\left[v_{H^{*}}, 1\right]$.

\section{Proof of Lemma 1}

First, note that $S_{y_{n}} \sim \operatorname{Poisson}\left(y_{n} \mu_{n}\right)$. Using the moment generating function for the Poisson,

$$
\begin{aligned}
\operatorname{Eexp}\left(\theta \frac{S_{y_{n}}}{y_{n}}\right) & =\operatorname{Eexp}\left(\frac{\theta}{y_{n}} S_{y_{n}}\right)=\exp \left(y_{n} \mu_{n}\left(e^{\theta / y_{n}}-1\right)\right) \\
& =\exp \left(y_{n} \mu_{n}\left(\frac{\theta}{y_{n}}+o\left(1 / y_{n}\right)\right)\right) \\
& =\exp \left(\mu_{n} \theta\right)+o(1)
\end{aligned}
$$

Hence,

$$
\lim _{n \rightarrow \infty} \mathrm{E} \exp \left(\theta S_{y_{n}} / y_{n}\right) \rightarrow \exp (\theta \mu)
$$

the moment generating function of the constant $\mu$. This guarantees convergence in distribution (see e.g. Billingsley[4, Section 30]).

\section{Proof of Theorem 2}

From the definitions of $\Lambda_{H}^{n}(x)$ and $\eta_{H}(x)$, and the relationship between $\lambda^{n}$ and $Q_{0}^{n}$, we have that

$$
\Lambda_{H}^{n}(x)=\frac{\eta_{H}(x)}{\rho^{n}} Q_{0}^{n} .
$$


Now, let $\left\{B_{i}\left(\eta_{H}(x)\left(\rho^{n}\right)^{-1}\right): i=1, \ldots, Q_{0}^{n}\right\}$ be a sequence of i.i.d Poisson r.v. with mean $\eta_{H}(x)\left(\rho^{n}\right)^{-1}$. It follows that $B\left(\Lambda_{H}^{n}(x)\right)$ has the same distribution as the sum of the $B_{i}\left(\eta_{H}(x)\left(\rho^{n}\right)^{-1}\right)$ from $i$ equals 1 to $Q_{0}^{n}$. Therefore, for a given $n$,

$$
\begin{aligned}
\mathbb{P}\left(P_{H}^{n}(v)<x\right) & =1, \quad \text { if } x>\hat{P} \text { or } \\
\mathbb{P}\left(P_{H}^{n}(v)<x\right) & =\mathbb{P}\left(B\left(\Lambda_{H}^{n}(x)\right) \leq Q_{0}^{n}-\mathbb{1}(x \leq v)\right) \\
& =\mathbb{P}\left(\sum_{i=1}^{Q_{0}^{n}} B_{i}\left(\eta_{H}(x)\left(\rho^{n}\right)^{-1}\right) \leq Q_{0}^{n}-\mathbb{1}(x \leq v)\right) \\
& =\mathbb{P}\left(\frac{\sum_{i=1}^{Q_{0}^{n}} B_{i}\left(\eta_{H}(x)\left(\rho^{n}\right)^{-1}\right)}{Q_{0}^{n}} \leq 1-\frac{\mathbb{1}(x \leq v)}{Q_{0}^{n}}\right), \quad \text { if } x \leq \hat{P} .
\end{aligned}
$$

Taking the LHS of the last inequality inside the parenthesis, we define

$$
\mathcal{B}^{n}(x) \triangleq \frac{\sum_{i=1}^{Q_{0}^{n}} B_{i}\left(\eta_{H}(x)\left(\rho^{n}\right)^{-1}\right)}{Q_{0}^{n}} .
$$

From Lemma $1, \mathcal{B}^{n}(x)$ converges in distribution to the constant $\eta_{H}(x) \rho^{-1}$. Moreover, it is clear that the RHS of the inequality in (30) converges to 1 . So, by focusing on the continuity points, the distribution of $P_{H}^{n}(v)$ converges weakly to the distribution:

$$
\mathbb{P}\left(P_{H}^{\infty}(v)<x\right)= \begin{cases}1 & \text { if } x>\hat{P} \\ 1 & \text { if } \rho>\eta_{H}(x) \text { and } x \leq \hat{P} \\ 0 & \text { if } \rho<\eta_{H}(x) \text { and } x \leq \hat{P}\end{cases}
$$

This corresponds to the distribution of the constant $\min \left\{\hat{P}, \eta_{H}^{-1}(\rho)\right\}$ at its continuity points, and so $\mathbb{P}\left(P_{H}^{n}(v)<x\right) \Rightarrow \mathbb{P}\left(P_{H}^{\infty}(v)<x\right)$. Thus, $P_{H}^{n}(v) \Rightarrow P_{H}^{\infty}(v)$, for $P_{H}^{\infty}(v)=\min \left\{\hat{P}, \eta_{H}^{-1}(\rho)\right\}$.

\section{Proof of Theorem 3}

From Theorem 2, we have that $P_{H^{*}}=\min \left\{v \in[0,1]: \eta_{H}(v) \leq \rho\right\}$. Since the function $\eta_{H}(v)$ is monotonically decreasing we conclude that $\eta_{H^{*}}\left(P_{H^{*}}\right)=\min \left\{\rho, \eta_{H^{*}}(0)\right\}$. This condition together with equation (19) and the equilibrium condition $H^{*}=\mathcal{R}\left(H^{*}\right)$ imply condition (20). The value of $v_{H^{*}}$ and $H^{*}$ follow from conditions (18) and (19), respectively.

\section{Proof of Theorem 4}

We first prove $K$-Lipschitz continuity of $\mathcal{R}(H)(v)$ in $(\tilde{v}, 1]$. Observe that due to the shape of the function $\mathcal{F}_{H}^{-1}(x)$ (flat at $T$ in the range $\left[0, \mathbb{P}\left(Q_{T}>0 \mid H\right)\right]$, and flat at zero for $x \geq \exp (w T)$ ), it is enough to prove this property in the range $\left(\mathbb{P}\left(Q_{T}>0 \mid H\right)\right.$, $\left.\exp (w T)\right)$, where $\mathcal{R}(H)(v)$ is differentiable. Let us define

$$
Z_{H}(v) \triangleq \frac{1}{v-\hat{P}} \int_{0}^{v} \mathbb{P}\left(P_{H}(v)<x\right) \mathrm{d} x
$$


Now, for any pair $v_{1}, v_{2} \in(\tilde{v}, 1]$ we have that

$$
\begin{aligned}
\left|\mathcal{R}(H)\left(v_{1}\right)-\mathcal{R}(H)\left(v_{2}\right)\right| & =\left|\mathcal{F}_{H}^{-1}\left(Z_{H}\left(v_{1}\right)\right)-\mathcal{F}_{H}^{-1}\left(Z_{H}\left(v_{2}\right)\right)\right| \\
& =\left|\int_{Z_{H}\left(v_{1}\right)}^{Z_{H}\left(v_{2}\right)} \frac{\mathrm{d}}{\mathrm{d} x} \mathcal{F}_{H}^{-1}(x) \mathrm{d} x\right|=\left|\int_{Z_{H}\left(v_{1}\right)}^{Z_{H}\left(v_{2}\right)}\left(\frac{\mathrm{d}}{\mathrm{d} \tau} \mathcal{F}_{H}(\tau)\right)^{-1}\right|_{\tau=\mathcal{F}_{H}^{-1}(x)} \mathrm{d} x \mid
\end{aligned}
$$

Note that the differentiability of $\mathcal{F}_{H}^{-1}(x)$ follows from the fact that the function $\mathcal{F}_{H}(\tau)$ is differentiable. In fact, from Lemma 2 we have that

$$
\frac{\mathrm{d}}{\mathrm{d} \tau} \mathcal{F}_{H}(\tau)=-\exp (w(T-\tau))\left[w \mathbb{P}\left(Q_{\tau}>0 \mid H\right)+\mathbb{P}\left(B\left(\Lambda_{H+}(\tau)\right)=Q_{0}-1\right) \frac{\mathrm{d}}{\mathrm{d} \tau} \Lambda_{H+}(\tau)\right],
$$

where

$$
\frac{\mathrm{d}}{\mathrm{d} \tau} \Lambda_{H+}(\tau)=\lambda \frac{\mathrm{d}}{\mathrm{d} \tau} \int_{0}^{1} \min \{\tau, H(v)\} \mathrm{d} F(v)=\lambda \int_{0}^{1} \mathbb{1}(\tau \leq H(v)) \mathrm{d} F(v) .
$$

Using the fact that $\mathbb{P}\left(Q_{\tau}>0 \mid H\right) \geq \mathbb{P}\left(Q_{T}>0 \mid H\right) \geq \mathbb{P}\left(B(\lambda T) \leq Q_{0}-1\right)$, that $0 \leq \frac{\mathrm{d}}{\mathrm{d} \tau} \Lambda_{H+}(\tau) \leq \lambda$, and that $1 \leq \exp (w(T-\tau)) \leq \exp (w T)$, we get that

$$
w \mathbb{P}\left(B(\lambda T) \leq Q_{0}-1\right) \leq\left|\frac{\mathrm{d}}{\mathrm{d} \tau} \mathcal{F}_{H}(\tau)\right| \leq \exp (w T)[w+\lambda]
$$

and so

$$
\left|\mathcal{R}(H)\left(v_{1}\right)-\mathcal{R}(H)\left(v_{2}\right)\right|=\left|\mathcal{F}_{H}^{-1}\left(Z_{H}\left(v_{1}\right)\right)-\mathcal{F}_{H}^{-1}\left(Z_{H}\left(v_{2}\right)\right)\right|=\leq\left(w \mathbb{P}\left(B(\lambda T) \leq Q_{0}-1\right)\right)^{-1}\left|Z_{H}\left(v_{1}\right)-Z_{H}\left(v_{2}\right)\right|
$$

$K$-Lipschitz continuity follows now combining this inequality and the following:

$$
\begin{aligned}
\left|Z_{H}\left(v_{1}\right)-Z_{H}\left(v_{2}\right)\right| & =\left|\int_{v_{1}}^{v_{2}} \frac{\mathrm{d}}{\mathrm{d} v} Z_{H}(v) \mathrm{d} v\right|=\left|\int_{v_{1}}^{v_{2}}\left[\frac{\mathbb{P}\left(P_{H}(v)<v\right)}{v-\hat{P}}-\int_{0}^{v} \frac{\mathbb{P}\left(P_{H}(v)<x\right)}{(v-\hat{P})^{2}} \mathrm{~d} x\right] \mathrm{d} v\right| \\
& \leq\left|\int_{v_{1}}^{v_{2}}\left[\frac{1}{\tilde{v}-\hat{P}}+\frac{1}{(\tilde{v}-\hat{P})^{2}}\right] \mathrm{d} x\right|=\left(\frac{1+\tilde{v}-\hat{P}}{(\tilde{v}-\hat{P})^{2}}\right)\left|v_{1}-v_{2}\right| .
\end{aligned}
$$

The constant $K$ equals $\left(w \mathbb{P}\left(B(\lambda T) \leq Q_{0}-1\right)\right)^{-1}\left(\frac{1+\tilde{v}-\hat{P}}{(\tilde{v}-\hat{P})^{2}}\right)$ and it is well-defined since $\tilde{v} \geq \underline{v}>\hat{P}$.

To prove the continuity in $\mathcal{H}$ of the mapping $\mathcal{R}$, we first note that the mapping $\mathcal{F}(H)$ is continuous in $\mathcal{H}$. In fact,

$$
\begin{aligned}
|\mathcal{F}(H)(\tau)-\mathcal{F}(\tilde{H})(\tau)| & =\exp (w(T-\tau))\left|\mathbb{P}\left(Q_{\tau}>0 \mid H\right)-\mathbb{P}\left(Q_{\tau}>0 \mid \tilde{H}\right)\right| \\
& =\exp (w(T-\tau))\left|\mathbb{P}\left(B\left(\Lambda_{H+}(\tau)\right) \leq Q_{0}-1\right)-\mathbb{P}\left(B\left(\Lambda_{\tilde{\mathrm{H}}+}(\tau)\right) \leq Q_{0}-1\right)\right| \\
& \leq \exp (w(T-\tau)) \mathbb{P}\left(B\left(Q_{0}-1\right)=Q_{0}-1\right)\left|\Lambda_{H+}(\tau)-\Lambda_{\tilde{\mathrm{H}}+}(\tau)\right| \\
& \leq \lambda \exp (w(T-\tau)) \mathbb{P}\left(B\left(Q_{0}-1\right)=Q_{0}-1\right)\|H-\tilde{H}\| \triangleq K_{\mathcal{F}}\|H-\tilde{H}\|,
\end{aligned}
$$

where the first inequality follows from Lemma 4, and the second one follows from the definition of $\Lambda_{H+}(\tau)$ and the property $|\min \{\tau, a\}-\min \{\tau, b\}| \leq|a-b|$. The continuity of the mapping $\mathcal{R}(H)=$ 
$\mathcal{F}(H)^{-1}$ follows now from

$$
\begin{aligned}
|\mathcal{R}(H)(v)-\mathcal{R}(\tilde{H})(v)|= & \left|\mathcal{F}(H)^{-1}\left(Z_{H}(v)\right)-\mathcal{F}(\tilde{H})^{-1}\left(Z_{\tilde{H}}(v)\right)\right| \\
\leq & \left|\mathcal{F}(H)^{-1}\left(Z_{H}(v)\right)-\mathcal{F}(\tilde{H})^{-1}\left(Z_{H}(v)\right)\right| \\
& +\left|\mathcal{F}(\tilde{H})^{-1}\left(Z_{H}(v)\right)-\mathcal{F}(\tilde{H})^{-1}\left(Z_{\tilde{H}}(v)\right)\right| .
\end{aligned}
$$

Regarding the first term in (33), from condition (32), we have that

$$
\left.\left|\mathcal{F}(\tilde{H})^{-1}\left(Z_{H}(v)\right)-\mathcal{F}(\tilde{H})^{-1}\left(Z_{\tilde{H}}(v)\right)\right| \leq\left(w \mathbb{P}\left(B(\lambda T) \leq Q_{0}-1\right)\right)^{-1} \mid Z_{H}(v)-Z_{\tilde{H}}(v)\right) \mid .
$$

As in the proof of Theorem 1, we can prove that

$$
\left.\mid Z_{H}(v)-Z_{\tilde{H}}(v)\right) \mid \leq K_{Z}\|H-\tilde{H}\|
$$

for an appropriate constant $K_{Z}$.

Now we focus on the second term in (33). Without loss of generality, suppose $\mathcal{F}(H)^{-1}\left(Z_{H}(v)\right) \leq$ $\mathcal{F}(\tilde{H})^{-1}\left(Z_{H}(v)\right)$. Using the continuity of $\mathcal{F}$ in $\mathcal{H}$ that we just proved, it follows that

$$
\mathcal{F}(\tilde{H})\left(\mathcal{F}(H)^{-1}\left(Z_{H}(v)\right)\right) \leq Z_{H}(v)+K_{\mathcal{F}}\|H-\tilde{H}\| .
$$

Applying $\mathcal{F}(H)^{-1}$ in both sides, and given that $\mathcal{F}(\tilde{H})^{-1}(v)$ is nonincreasing in $v$, we have that

$$
\mathcal{F}(H)^{-1}\left(Z_{H}(v)\right)=\mathcal{F}(\tilde{H})^{-1}\left(\mathcal{F}(\tilde{H})\left(\mathcal{F}(H)^{-1}\left(Z_{H}(v)\right)\right) \geq \mathcal{F}(\tilde{H})^{-1}\left(Z_{H}(v)+K_{\mathcal{F}}\|H-\tilde{H}\|\right) .\right.
$$

From the assumption $\mathcal{F}(H)^{-1}\left(Z_{H}(v)\right) \leq \mathcal{F}(\tilde{H})^{-1}\left(Z_{H}(v)\right)$ we get

$$
\begin{aligned}
\left|\mathcal{F}(H)^{-1}\left(Z_{H}(v)\right)-\mathcal{F}(\tilde{H})^{-1}\left(Z_{H}(v)\right)\right| & \leq\left|\mathcal{F}(\tilde{H})^{-1}\left(Z_{H}(v)+K_{\mathcal{F}}\|H-\tilde{H}\|\right)-\mathcal{F}(\tilde{H})^{-1}\left(Z_{H}(v)\right)\right| \\
& \leq\left(w \mathbb{P}\left(B(\lambda T) \leq Q_{0}-1\right)\right)^{-1} K_{\mathcal{F}}\|H-\tilde{H}\|,
\end{aligned}
$$

where the second inequality follows from condition (32) above.

Therefore, using the bounds for the two absolute terms in (33), we conclude that

$$
|\mathcal{R}(H)(v)-\mathcal{R}(\tilde{H})(v)| \leq\left(w \mathbb{P}\left(B(\lambda T) \leq Q_{0}-1\right)\right)^{-1}\left(K_{Z}+K_{\mathcal{F}}\right)\|H-\tilde{H}\|,
$$

which proves the continuity of $\mathcal{R}$ in $\mathcal{H}$.

Finally, as in Theorem 1, the existence of a symmetric equilibrium follows again from the SchauderTychonoff Fixed-Point Theorem.

\section{Proof of Theorem 5}

Recall that $B\left(\Lambda_{H-}^{n}(x)\right)$ is a Poisson r.v. with mean $\Lambda_{H-}^{n}(x)$. To prove $i$ ), we start by rewriting $\Lambda_{H-}^{n}(x)$ as

$$
\Lambda_{H-}^{n}(x)=\frac{\eta_{H-}(x)}{\rho^{n}} Q_{0}^{n}
$$


Let $\left\{B_{i}\left(\eta_{H_{-}}(x)\left(\rho^{n}\right)^{-1}\right): i=1, \ldots, Q_{0}^{n}\right\}$ be a sequence of i.i.d Poisson random variables with mean $\eta_{H_{-}}(x)\left(\rho^{n}\right)^{-1}$. The random variable $B\left(\Lambda_{H_{-}}^{n}(x)\right)$ has the same distribution as the sum of the $B_{i}\left(\eta_{H_{-}}(x)\left(\rho^{n}\right)^{-1}\right)$, $1 \leq i \leq Q_{0}^{n}$. For a fixed $0 \leq \alpha \leq 1$,

$$
\begin{aligned}
\mathbb{P}\left(Q_{T}^{n} \geq \alpha Q_{0}^{n}\right) & =\mathbb{P}\left(B\left(\Lambda_{H-}^{n}(0)\right) \leq Q_{0}^{n}(1-\alpha)\right) \\
& =\mathbb{P}\left(\sum_{i=1}^{Q_{0}^{n}} B_{i}\left(\eta_{H-}(0)\left(\rho^{n}\right)^{-1}\right) \leq Q_{0}^{n}(1-\alpha)\right) \\
& =\mathbb{P}\left(\frac{\sum_{i=1}^{Q_{0}^{n}} B_{i}\left(\eta_{H-}(0)\left(\rho^{n}\right)^{-1}\right)}{Q_{0}^{n}} \leq 1-\alpha\right),
\end{aligned}
$$

where the first equality follows from the fact that all the units put to the auction are the remaining ones from the list price channel. Let

$$
\mathcal{B}_{-}^{n}(0) \triangleq \frac{\sum_{i=1}^{Q_{0}^{n}} B_{i}\left(\eta_{H-}(0)\left(\rho^{n}\right)^{-1}\right)}{Q_{0}^{n}} .
$$

From Lemma $1, \mathcal{B}_{-}^{n}(0)$ converges in distribution to the constant $\eta_{H_{-}}(0) \rho^{-1}$. Given that for $n$ sufficiently big, $Q_{0}^{n}=n Q_{0}+o(n)$, then by focusing on the continuity points, the tail distribution of $Q_{T}^{n} / n$ converges weakly to the tail distribution:

$$
\bar{F}_{Q_{T}}\left(\alpha Q_{0}\right)= \begin{cases}1 & \text { if } \eta_{H-}(0)<\rho(1-\alpha) \\ 0 & \text { if } \eta_{H-}(0)>\rho(1-\alpha)\end{cases}
$$

In words, the first case corresponds to $\alpha Q_{0}<Q_{0}-\lambda T \eta_{H_{-}}(0)$, that is, there are more units available in the auction channel than the requested $\alpha Q_{0}$; the second case is the opposite. This is the distribution of the constant $\left(Q_{0}-\lambda T \eta_{H_{-}}(0)\right)^{+}$at its continuity points, and so $Q_{T}^{n} / n \Rightarrow Q_{T}$.

For part $i i)$, we have:

$$
\begin{aligned}
\mathbb{P}\left(P_{H}^{n}(v)<x\right) & =\mathbb{P}\left(B\left(\Lambda_{H}^{n}(x)\right) \leq Q_{T}^{n}-\mathbb{1}(x \leq v)\right) \\
& =\mathbb{P}\left(\sum_{i=1}^{Q_{0}^{n}} B_{i}\left(\eta_{H}(x)\left(\rho^{n}\right)^{-1}\right) \leq Q_{T}^{n}-\mathbb{1}(x \leq v)\right) \\
& =\mathbb{P}\left(\frac{\sum_{i=1}^{Q_{0}^{n}} B_{i}\left(\eta_{H}(x)\left(\rho^{n}\right)^{-1}\right)}{Q_{0}^{n}} \leq \frac{Q_{T}^{n}-\mathbb{1}(x \leq v)}{Q_{0}^{n}}\right)
\end{aligned}
$$

A similar argument to the one above shows that as $n \rightarrow \infty$,

$$
\mathcal{B}^{n}(x) \triangleq \frac{\sum_{i=1}^{Q_{0}^{n}} B_{i}\left(\eta_{H}(x)\left(\rho^{n}\right)^{-1}\right)}{Q_{0}^{n}} \Rightarrow \eta_{H}(x) \rho^{-1}
$$

Regarding the RHS in (34), from part $i$ ) if a final auction occurs, for $n$ large enough we have $Q_{T}^{n} \approx$ $Q_{0}^{n}-\lambda^{n} T \eta_{H-}(0)$. Then, as $n \rightarrow \infty$,

$$
\frac{Q_{T}^{n}-\mathbb{1}(x \leq v)}{Q_{0}^{n}} \rightarrow 1-\frac{\eta_{H-}(0)}{\rho} .
$$


By focusing on the continuity points, the distribution of $P_{H}^{n}(v)$ converges weakly to the distribution:

$$
\mathbb{P}\left(P_{H}^{\infty}(v)<x\right)= \begin{cases}1 & \text { if } \eta_{H}(x)<\rho-\eta_{H-}(0) \\ 0 & \text { if } \eta_{H}(x)>\rho-\eta_{H-}(0)\end{cases}
$$

This corresponds to the distribution of the constant $P_{H}^{\infty}=\min \left\{v \in[0,1]: \eta_{H}(v) \leq \rho-\eta_{H-}(0)\right\}$ at its continuity points, and so $P_{H}^{n}(v) \Rightarrow P_{H}^{\infty}$.

\section{References}

[1] C. Beam and A. Segev. Auction on the Internet: A field study. Working paper 1032, Haas School of Business, University of California at Berkeley, 1998.

[2] P.C. Bell. Revenue management: That's the ticket. OR/MS Today, 25(2), April 1998.

[3] D. Berstsekas and J. Tsitsiklis. Neuro-Dynamic Programming. Athena Scientific, Belmont, MA, 1996.

[4] P. Billingsley. Probability and Measure. John Wiley \& Sons, New York, NY, 1995.

[5] G. Bitran and R. Caldentey. An overview of pricing models for revenue management. MESOM, $5,2003$.

[6] E. A. Boyd and I. Bilegan. Revenue management and E-Commerce. Management Science, 49:1363-1386, 2003.

[7] E. Budish and L. Takeyama. Buy price in online auctions: Irrationality on the Internet? Economic Letters, 72:325-333, 2001.

[8] J. Bulow and J. Roberts. The simple economics of optimal auctions. Journal of political economy, 97:1060-1090, 1989.

[9] W. Cheney. Analysis for Applied Mathematics. Springer-Verlag, New York, NY, 2001.

[10] W.L. Cooper and R.P. Menich. Airline ticket auctions: Revenue management and the pivotal mechanism. Working paper, School of Industrial and Systems Engineering, Georgia Institute of Technology, Atlanta, GA, 1998.

[11] R. Durrett. Probability: Theory and Examples. Duxbury Press, Belmont, CA, 1996.

[12] H. Etzion, E. Pinker, and A. Seidmann. Analyzing the simultaneous use of auctions and posted prices for on-line selling. Working paper CIS-03-01, William E. Simon Graduate School of Business Administration, University of Rochester, 2003.

[13] J. Gallien. Dynamic mechanism design for online commerce. MIT Working paper 4268-02, 2002. 
[14] A. Glazer and R. Hassin. ?/m/1: On the equilibrium distribution of customer arrivals. European Journal of Operational Research, 13:146-150, 1983.

[15] R. Harstad. Alternative common-value auction procedures: Revenue comparisons with free entry. Journal of Political Economy, 98:421-429, 1990.

[16] S. Klein. Introduction to electronic auctions. Electronic Markets, 7:3-6, 1997.

[17] S. Klein and R. O'Keefe. The impact of the Web on auctions: Some empirical evidence and theoretical considerations. International Journal of Electronic Commerce, 3:7-20, 1999.

[18] P. Klemperer. Auction theory: A guide to the literature. Journal of Economic Surveys, 13:227286, 1999.

[19] V. Krishna. Auction theory. Academic Press, New York, NY, 2002.

[20] M. Lariviere and J. Van Mieghem. Strategically seeking service: How competition can generate poisson arrivals. Manufacturing and Service Operations Management, 6:23-40, 2003.

[21] D. Lucking-Reiley. Using field experiments to test equivalence between auction formats: Magic on the Internet. American Economic Review, 89:1063-1080, 1999.

[22] D. Lucking-Reiley. Auctions on the Internet: What's been auctioned, and how? Journal of Industrial Economics, 48:227-252, 2000.

[23] A. Mas-Colell, M. Whinston, and J. Green. Microeconomic Theory. Oxford University Press, New York, NY, 1995.

[24] E. Maskin and J. Riley. Optimal multi-unit auctions. In F. Hahn, editor, The Economics of Missing Markets, Information and Games, pages 312-336. Oxford University Press, 1989.

[25] P. Milgrom and R. Weber. A theory of auctions and competitive bidding. Econometrica, 50:10891122, 1982.

[26] R. Myerson. Optimal auction design. Mathematics of Operations Research, 6:58-73, 1981.

[27] E. Pinker, A. Seidmann, and Y. Vakrat. Using transaction data for the design of sequential, multi-unit, online auctions. Working Paper CIS-00-03, William E. Simon Graduate School of Business Administration, University of Rochester, 2000.

[28] E. Pinker, A. Seidmann, and Y. Vakrat. Managing online auctions: Current business and research issues. Management Science, 49:1457-1484, 2003.

[29] J. Riley and W. Samuelson. Optimal auctions. The American Economic Review, 71:381-392, 1981. 
[30] A. Roth and A. Ockenfels. Last minute bidding and the rules for ending second-price auctions: Evidence from eBay and Amazon auctions on the Internet. American Economic Review, 92:10931103, 2002.

[31] M.H. Rothkopf and R.M. Harstad. Modeling competitive bidding: A critical essay. Management Science, 40:364-384, 1994.

[32] K. Talluri and G. van Ryzin. The theory and practice of revenue management. Kluwer Academic Press, Boston, MA, 2004.

[33] Y. Vakrat and A. Seidmann. Can online auctions beat online catalogs? In Proceedings of the 20th International Conference on Information Systems, Charlotte, NC, 1999.

[34] G.J. van Ryzin and G. Vulcano. Optimal auctioning and ordering in an infinite horizon inventorypricing system. Forthcoming in Operations Research, Vol. 52(3), 2004.

[35] A. De Vany. Institutions for stochastic markets. Journal of Institutional and Theoretical Economics, 143:91-103, 1987.

[36] W. Vickrey. Counterspeculation, auctions and competitive sealed tenders. Journal of Finance, $16: 8-37,1961$.

[37] G. Vulcano, G.J. van Ryzin, and C. Maglaras. Optimal dynamic auctions for revenue management. Management Science, 48:1388-1407, 2002.

[38] W. Wang. Auctions versus posted-price selling. American Economic Review, 83:838-851, 1993. 